\title{
The particle spectrum of the Tricritical Ising Model with spin reversal symmetric perturbations
}

\author{
L Lepori ${ }^{1,2}$, G Mussardo ${ }^{1,2,3}$, G Zs Tóth ${ }^{1,2,4}$ \\ ${ }^{1}$ International School for Advanced Studies (SISSA), Via Beirut 2-4, 34014 Trieste, \\ Italy \\ ${ }^{2}$ INFN Sezione di Trieste, Italy \\ 3 The Abdus Salam International Centre for Theoretical Physics, Trieste, Italy \\ ${ }^{4}$ Research Institute for Particle and Nuclear Physics, Hungarian Academy of Sciences, \\ Pf. 49, 1525 Budapest, Hungary \\ E-mail: lepori@sissa.it, mussardo@sissa.it, toth@sissa.it and \\ tgzs@cs.elte.hu
}

\begin{abstract}
We analyze the evolution of the particle spectrum of the Tricritical Ising Model by varying the couplings of the energy and vacancy density fields. The particle content changes from the spectrum of a supersymmetric theory (either of an exact or a spontaneously broken supersymmetric theory) to the spectrum of seven particles related to the underlying $E_{7}$ structure. In the low temperature phase some of these excitations are topologically charged particles that are stable under an arbitrary variation of the parameters. The high and low temperature phases of the model are related by duality. In some regions of the two couplings there are also present false vacua and sequences of bound states. In order to study the non-integrable features of this model we employ the Form Factor Perturbation Theory and the Truncated Conformal Space Approach.
\end{abstract}

This paper is dedicated to the memory of Aliosha Zamolodchikov. 


\section{Introduction}

The two-dimensional Tricritical Ising Model (TIM), which is the second in the unitary series of conformal minimal models after the critical Ising model [1], describes critical phenomena in a variety of systems with tricritical points. It also exhibits superconformal symmetry, being the first member of the series of superconformal minimal models [2], and it has interesting symmetries related to the coset constructions $s u(2)_{2} \otimes s u(2)_{1} / s u(2)_{3}$ and $e(7)_{1} \otimes e(7)_{1} / e(7)[3,4$. Due to these properties the TIM has attracted interest for many years.

In the present paper we study the particle spectrum of the TIM perturbed by those relevant scaling fields which are invariant under spin reversal. These fields are the energy density $\varepsilon$ and the vacancy density $t$, with conformal weights given by $\Delta_{2}=1 / 10$ and $\Delta_{4}=3 / 5$ respectively 1 . The action of the perturbed model can formally be written as

$$
\mathcal{A}=\mathcal{A}_{0}+g_{2} \int \varepsilon(z, \bar{z}) \mathrm{d}^{2} z+g_{4} \int t(z, \bar{z}) \mathrm{d}^{2} z,
$$

where $\mathcal{A}_{0}$ is the action of the TIM at its conformal point, whereas $g_{2}$ and $g_{4}$ are the two coupling constants of our theory. Their dimensions are fixed by the conformal weights of the conjugate fields, $g_{i} \sim \mathcal{M}^{2-2 \Delta_{i}}$, where $\mathcal{M}$ is an arbitrary mass scale of the off-critical model. The ratios of the particle masses of the model (1.1), being universal quantities, depend only on a single dimensionless combination $\eta$ of the two coupling constants $g_{2}$ and $g_{4}$, that we choose as

$$
\eta=\frac{g_{4}}{\left|g_{2}\right|^{\frac{2-2 \Delta_{4}}{2-2 \Delta_{2}}}}=\frac{g_{4}}{\left|g_{2}\right|^{\frac{4}{9}}} .
$$

In the following we write $\eta_{+}$if $g_{2}>0$ and $\eta_{-}$if $g_{2}<0$.

The evolution of the particle spectrum by varying $\eta$ is an important characteristic of the class of universality associated with the TIM. It is worth stressing that the action (1.1) provides the simplest example of a bosonic theory having kinks in its spectrum which do not get confined under continuous variations of the coupling constants. To appreciate this feature, one has to recall that, in a generic bosonic theory, the kinks are quite fragile objects: they get generally confined under a small perturbation of the parameters (see, for instance, refs. [6, [7]). The stability of the kinks can be guaranteed, for instance, by supersymmetry but in theories that have also fermions [9]. In purely bosonic theories, the robustness of the kink states may only come via a fine-tuning mechanism or special symmetry of the perturbing operators. In the model analyzed in this paper, the latter property is the spin reversal invariance of both perturbations. The study of the particle spectrum of this model is, in any case, interesting in itself, since the action (1.1) is nonintegrable if both $g_{2}$ and $g_{4}$ are non-zero.

${ }^{1}$ For further details on the model and its symmetry we refer the reader to the article 5 . 


\section{A quick glimpse into the model}

In the TIM, the parameter $g_{2}$ can be interpreted as the difference between the actual temperature and its critical value. The $g_{2}=0$ critical line divides the phase plane into the high temperature $\left(g_{2}>0\right)$ and the low temperature $\left(g_{2}<0\right)$ halves, which are mapped into each other by a duality transformation under which $\left(g_{2}, g_{4}\right) \leftrightarrow\left(-g_{2}, g_{4}\right)$. Along the critical line $g_{2}=0$, the model (1.1) is integrable and has also supersymmetry [10, 11, 12]: the $g_{4}>0$ half line consists of a massless renormalization group flow to the Ising critical point in which the supersymmetry is spontaneously broken, while the $g_{4}<0$ half line consists of first order phase transition points with unbroken supersymmetry. The meeting point of these half lines is the tricritical point. The model is also integrable along the $g_{4}=0$ line where its particle spectrum and S-matrix are related to the $E_{7}$ Lie-algebra [13, 14], therefore we shall refer to this line as the $E_{7}$-related line.

It is well known that the $\phi^{6}$ Landau-Ginzburg theory has a tricritical point which is described by the TIM [15], in particular the model (1.1) admits another description in terms of the action

$$
\mathcal{A}_{L G}=\int \mathrm{d}^{2} x\left(\frac{1}{2}\left(\partial_{\mu} \phi\right)^{2}+\phi^{6}+a_{2} \phi^{2}+a_{4} \phi^{4}\right),
$$

with the identification $\varepsilon \sim: \phi^{2}:, t \sim: \phi^{4}:$ In this formulation the tricritical point corresponds to $a_{2}=a_{4}=0$; the spin reversal transformation acts on $\phi$ as $\phi \mapsto-\phi$. This correspondence allows one to associate the Landau-Ginzburg potentials with the various parts of the $\left(g_{2}, g_{4}\right)$ phase plane, as shown in Figure 1

The shape of the potentials in Figure 1 provides a useful guide to the nature of the excitations at the various points of the $\left(g_{2}, g_{4}\right)$ plane. For instance, in the high temperature phase, where the spin reversal symmetry is unbroken, the potential has a unique vacuum and therefore the particles do not have topological charge in this phase. Moving up from the positive horizontal axis in the first quadrant, the curvature of the minimum decreases until it vanishes when we reach the positive vertical axis: the theory has a massless spectrum at this point. Instead, moving down from the positive horizontal axis in the forth quadrant, two metastable vacua start to appear and they become abruptly degenerate with the true vacuum once the negative vertical axis is reached. Right at this point the theory has kink excitations, i.e. topologically charged particles, that interpolate between the three vacua.

In the low temperature phase, i.e. in the second and third quadrants, the spin reversal symmetry is instead spontaneously broken and the ground state is doubly degenerate, hence in these regions we expect that the spectrum consists of kinks and possible bound states thereof. Moreover, it is sufficient to look at the evolution of the potential in these quadrants in order to conclude that the kinks of this model are always stable excitations (at least those with lowest mass), no matter how we vary the coupling constants. In more detail, starting from the negative horizontal axis and moving up in the third quadrant, the barrier between the two vacua decreases until it disappears when we reach the positive vertical axis. Hence, we expect the masses of the kinks and their possible bound states 
to decrease along this trajectory so that they adiabatically vanish once we arrive at the positive vertical axis. On the other hand, moving down from the negative horizontal axis in the third quadrant, a metastable vacuum starts to appear in the middle of the barrier: this state becomes abruptly degenerate with the two existing vacua just when we reach the negative vertical axis. At this point, the kink excitations between the two original vacua break down and give rise to two different sets of topological excitations relative to the new threefold vacuum structure.

In the following we are going to support the physical scenario described above by using a combination of analytic and numerical methods. The first method is the Form Factor Perturbation Theory (FFPT) [6] that allows us to get analytic information on the spectrum in the vicinity of the integrable lines. The second method is the Truncated Conformal Space Approach (TCSA) [18], which is one of the best suited numerical approaches for extracting the spectrum of perturbed conformal field theories. The spin reversal and the duality symmetries of the TIM play also an important role in the implementation of both methods. Let's finally mention that the two integrable cases $g_{2}=0$ and $g_{4}=0$ were studied by TCSA in [5]; the present work can thus be regarded as an extension of those studies to the non-integrable $\left(g_{2} \neq 0, g_{4} \neq 0\right)$ domain.

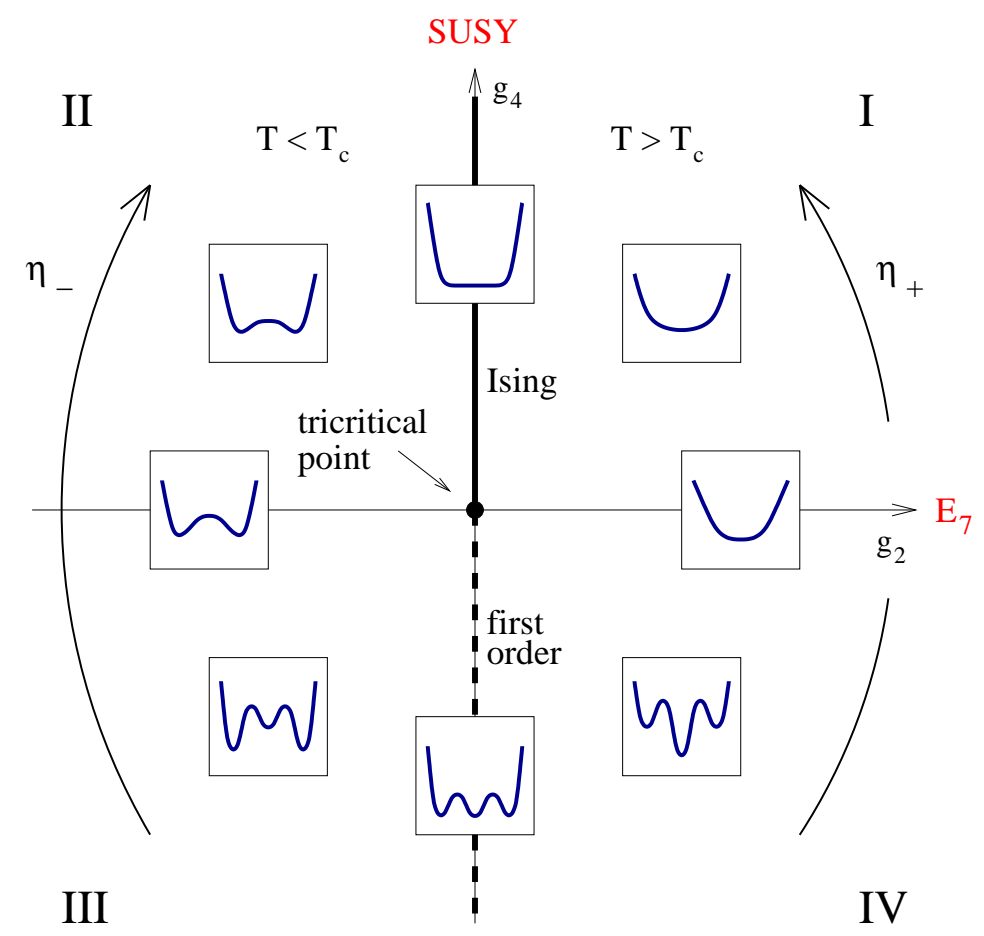

Figure 1. Landau-Ginzburg potentials associated with the various parts of the phase space.

\section{The two methods}

In this section we briefly discuss the two techniques that have been employed to study the evolution of the spectrum of our model. 


\subsection{Form Factor Perturbation Theory}

The Form Factor Perturbation Theory permits to investigate the behaviour of a theory in the vicinity of an integrable direction, either if the integrable theory is massive [6, 7] or massless [8]. Let $\mathcal{A}_{\text {int }}$ be the action of the integrable model and $\Upsilon(x)$ the field that moves the system away from integrability, so that nearby the action of the theory can be written as

$$
\mathcal{A}=\mathcal{A}_{\text {int }}+\lambda \int \mathrm{d}^{2} x \Upsilon(x)
$$

If the original integrable model has scalar excitations $2 A_{1}(\theta), A_{2}(\theta), \ldots$, the first order correction in $\lambda$ to their mass $M_{k}$ is given by

$$
\delta M_{k}^{2} \simeq 2 \lambda F_{k}^{\Upsilon}(\mathrm{i} \pi)
$$

where

$$
F_{k}^{\Upsilon}\left(\theta_{12}\right) \equiv\left\langle 0|\Upsilon(0)| A_{k}\left(\theta_{1}\right) A_{k}\left(\theta_{2}\right)\right\rangle
$$

is the two-particle form factor of the operator $\Upsilon(x)$, with $\theta_{12}=\theta_{1}-\theta_{2}$. A similar formula also holds if the integrable model has massless excitations, with a proper interpretation of the rapidity variable $\theta$ [8]. If, instead, the integrable theory has topological kinks $\left|K_{a b}(\theta)\right\rangle$ interpolating between the vacuum states $|a\rangle$ and $|b\rangle$, the relevant formula is

$$
\delta M_{a b}^{2} \simeq 2 \lambda F_{a b}^{\Upsilon}(\mathrm{i} \pi)
$$

where

$$
F_{a b}^{\Upsilon}\left(\theta_{12}\right) \equiv{ }_{k}\left\langle 0|\Upsilon(0)| K_{a b}\left(\theta_{1}\right) K_{b a}\left(\theta_{2}\right)\right\rangle .
$$

In integrable field theories the form factors of a generic scalar operator $\Upsilon(x)$ can be computed exactly [16, 17] and therefore we can study the evolution of the spectrum near to the integrable lines. However, one has to be careful when applying the formulas (3.3) and (3.5) since the two-particle form factor can be singular at $\theta_{12}=\mathrm{i} \pi$. This happens if the perturbing operator $\Upsilon(x)$ has a semi-local index $\gamma$ with respect to the particle excitations (this is usually the case of the topological kinks, for instance). In this case the point $\theta_{12}= \pm \mathrm{i} \pi$ is the location of a simple pole of the two-particle form factor, whose residue is given by [7, 17]

$$
-\mathrm{i} \operatorname{Res}_{\theta_{12}= \pm \mathrm{i} \pi} F_{a b}^{\Upsilon}\left(\theta_{12}\right)=\left(1-\mathrm{e}^{\mp 2 \mathrm{i} \pi \gamma}\right)_{k}\langle 0|\Upsilon| 0\rangle_{k} .
$$

The result of the brief analysis given above can be summarized as follows. If $F_{l}^{\Upsilon}$ (i $\pi$ ) is finite, the corresponding particle or kink excitation survives the perturbation and its mass is adiabatically shifted from the original value. Vice versa, if $F_{l}^{\Upsilon}(\mathrm{i} \pi)$ is divergent, the corresponding excitation no longer survives as asymptotic particle of the perturbed theory, i.e. it gets confined.

2 The rapidity variable $\theta$ expresses the dispersion relation of the excitations. If we consider a massive particle of mass $m$, its energy and momentum are given by $E=m \cosh \theta, p=m \sinh \theta$. If, instead, the particle is massless, it can be a right or a left mover excitation. The dispersion relations of a right mover are $E_{R}=p=M \mathrm{e}^{\theta}$, while those of a left mover $E_{L}=-p=M \mathrm{e}^{-\theta}$, where $M$ is an arbitrary mass scale. 


\subsection{The truncation method}

The Truncated Conformal Space Approach (TCSA) [18] can be used to study perturbed conformal field theories. It consists in a finite size analysis of the spectrum of a theory on a cylinder of circumference $R$. This is realized by truncating the conformal basis at a certain level $n$ in the Verma modules of the irreducible representations of the primary fields and solving the eigenvalue and eigenvector problem of the truncated Hamiltonian operator numerically.

To extract a quantity $F$ relative to the $R \rightarrow \infty$ limiting model, one calculates $F(R, n)$ (i.e. $F$ at a cylinder circumference $R$ and truncation level $n$ ) for several values of $R$, and possibly also for several values of $n$. Usually there is a range $R_{F, \min }<R<R_{F, \max }(n)$ such that in this range $F(R, n)$ is an approximately constant function of $R$, and its value can be regarded as a good approximation of the infinite-volume value of $F$. This range is called the physical window for $F$. Both $R_{F, \min }$ and $R_{F, \max }(n)$ depend on $F$, and $R_{F, \max }(n)$ also depends on $n$ and is expected to tend to infinity as $n$ is increased. Hence, the approximation to $F$ usually improves if $n$ is increased. For $R>R_{F, \max }(n)$ the truncation effects spoil the approximation to $F$ and give rise to an unphysical window of values for this quantity.

We determine the masses of the particles from the energy differences

$$
\Delta E_{i}(R, n) \equiv\left(E_{i}(R, n)-E_{0}(R, n)\right)
$$

where $E_{0}(R, n)$ is the lowest energy line. In our case, the individual energy levels $E_{i}(R, n)$ diverge as $n \rightarrow \infty$ if $g_{4} \neq 0$, since the conformal weight of the perturbation $t$ is greater than $1 / 2$, the energy differences $E_{i}(R, n)-E_{0}(R, n)$ are however convergent [19]. We also use the TCSA to calculate form factors and vacuum expectation values. This can be done by computing numerically the eigenvectors belonging to the various energy eigenvalues.

The Hamiltonian operator of the model (1.1) on a cylinder of circumference $R$ is

$$
\begin{aligned}
H=\frac{2 \pi}{R}\left(L_{0}\right. & \left.+\bar{L}_{0}-\frac{c}{12}\right) \\
& +2 \pi g_{2} \lambda_{2} R^{-2 \Delta_{2}} \int_{0}^{R} \varepsilon(x, 0) \mathrm{d} x+2 \pi g_{4} \lambda_{4} R^{-2 \Delta_{4}} \int_{0}^{R} t(x, 0) \mathrm{d} x,
\end{aligned}
$$

where $c=7 / 10$ is the central charge of the TIM, whereas $L_{0}$ and $\bar{L}_{0}$ are the zero index generators of the chiral Virasoro algebras. For convenience, we have chosen to introduce the quantities

$$
\lambda_{2}=(2 \pi)^{2 \Delta_{2}-1} \times 0.0928344, \quad \lambda_{4}=(2 \pi)^{2 \Delta_{4}-1} \times 0.1486960 .
$$

These are precisely the values of the coupling constants that ensure a mass gap $M=1$ when we specialize the above Hamiltonian to the integrable massive cases $\left(g_{2}= \pm 1, g_{4}=\right.$ $0)$ and $\left(g_{2}=0, g_{4}=-1\right)$ [20]. Further data which are necessary to implement the TCSA for the TIM can be found in [5].

To study the mass spectrum it is sufficient to consider the zero momentum subspace

only. Moreover, one can also treat the parts of the Hamiltonian which are even or odd 
with respect to spin reversal separately 3 . In our calculation we used periodic boundary conditions, which selects the sector of zero topological charge in the Hilbert space of the multi-particle states. As we will show below, there may be other phenomena related to the boundary conditions and to the finite size of the system that have to be properly taken into account in order to interpret the spectrum correctly.

In our numerical calculations we varied the coupling constants $g_{2}$ and $g_{4}$ along the square with corners $( \pm 1, \pm 1)$ shown in Figure 2. The truncation level that we used is $n=8$, that corresponds to a Hamiltonian truncated to 1624 conformal states. The finite volume spectra shown in Figures 4, 8, 9 and 10 are always plotted as functions of $m R$, where $m$ is the mass gap, whose value depends however on the region of the coupling constants which each figure refers to.

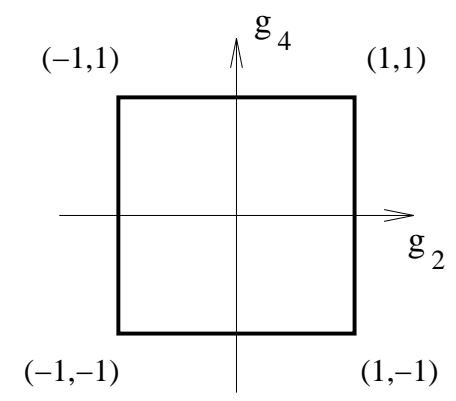

Figure 2. The square in the $\left(g_{2}, g_{4}\right)$ plane along which the TCSA calculations were done.

\section{The spectrum in the high temperature phase}

In this section we describe our results on the particle spectrum of the model (1.1) in the high temperature phase, made up of the first and the fourth quadrant of the $\left(g_{2}, g_{4}\right)$ plane. This phase is described by the variable $\eta_{+} \in(-\infty,+\infty)$ : the value $\eta_{+}=-\infty$ corresponds to the negative vertical axis, $\eta_{+}=0$ to the positive horizontal axis, and $\eta_{+}=+\infty$ to the positive vertical axis.

In this phase the $\mathbb{Z}_{2}$ spin reversal symmetry is exact and there is a unique vacuum of the theory (although in the fourth quadrant there are also two false vacua). Hence here the excitations are ordinary scalar particles. In order to follow the evolution of the spectrum it is convenient to start our analysis from the vicinity of the negative vertical axis, i.e. from the fourth quadrant.

3 In addition to the two spin reversal even fields $\varepsilon$ and $t$, the TIM possesses other two even fields, the identity operator $I$ and the supersymmetry generator $G$, with conformal weights 0 and $3 / 2$ respectively. The spin reversal odd sector is generated by the magnetization operator $\sigma$ and the subleading magnetization $\sigma^{\prime}$, with $\Delta_{\sigma}=3 / 80$ and $\Delta_{\sigma^{\prime}}=7 / 16$. 
The particle spectrum of the TIM with spin reversal symmetric perturbations

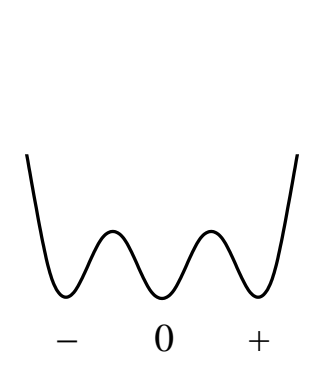

(a)

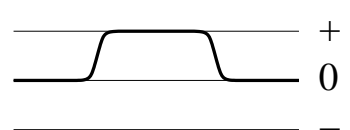

(i)

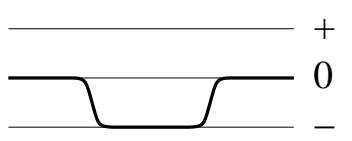

(iii)

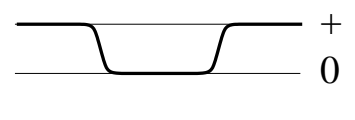

(ii)

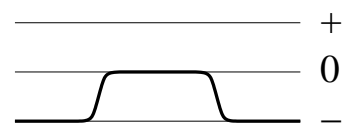

(iv)

(b)

Figure 3. (a) The three vacua in the first-order transition point. (b) Unbound kinkantikink pairs. On the cylinder with periodic boundary the configurations (i) and (ii) represent the same state, and so do (iii) and (iv).

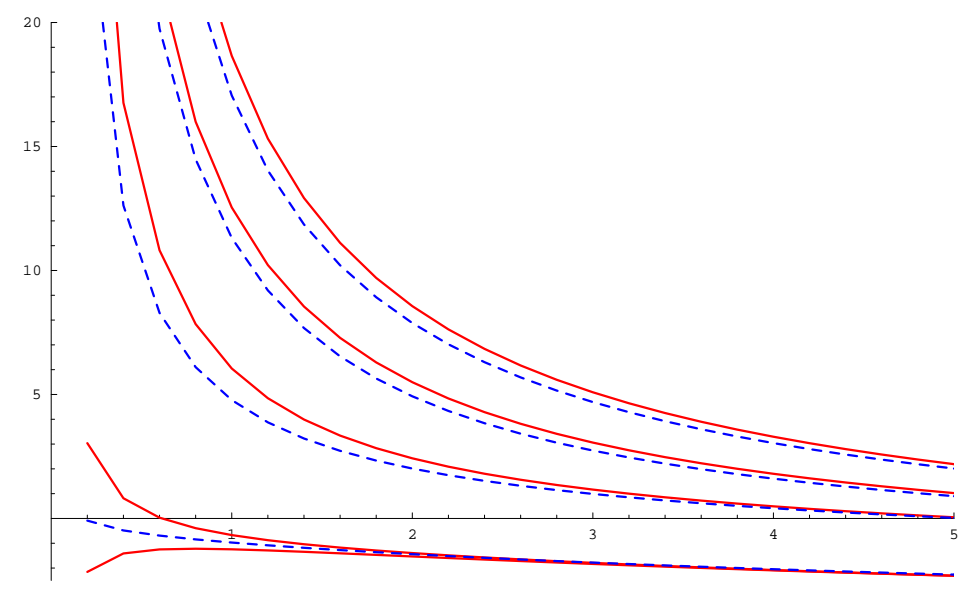

Figure 4. The lowest nine energy levels $E_{i}, i=0 \ldots 8$ in the first-order transition point as functions of $m R$, obtained by TCSA. Red lines are even, blue (dashed) lines are odd under spin reversal. $m$ denotes the mass gap.

\subsection{The fourth quadrant}

Precisely at $\eta_{+}=-\infty$, the model has a first order phase transition. This means that along the negative vertical axis the theory has three degenerate ground states $|0\rangle,|+\rangle$ and $|-\rangle$ : the vacuum $|0\rangle$ is even under the spin reversal symmetry $Q$, while $|+\rangle$ and $|-\rangle$ are mapped into each other: $|-\rangle=Q|+\rangle$ (see Figure 3. a). Right at $\eta_{+}=-\infty$, the theory presents also an exact supersymmetry: the particle spectrum consists of four kinks $\left|K_{-0}\right\rangle,\left|K_{0-}\right\rangle,\left|K_{0+}\right\rangle$ and $\left|K_{+0}\right\rangle$ of equal mass. These kinks, that do not have bound states, provide an irreducible representation of supersymmetry and their exact S-matrix was obtained in [11]. The action of the spin reversal symmetry $Q$ on the kinks is given by $Q\left|K_{-0}\right\rangle=\left|K_{+0}\right\rangle, Q\left|K_{0-}\right\rangle=\left|K_{0+}\right\rangle$.

On a cylinder of circumference $R$, the three degenerate vacua give rise to three exponentially split energy lines, for the phenomenon of tunneling that occurs at a finite volume. With periodic boundary conditions, there are however no energy lines corresponding to the one-particle states of the kinks. Above the three exponentially 
degenerate lines of the ground states, one finds instead doublets of lines corresponding to the neutral kink-antikink configurations (see Figure 4). The proper counting of the states appearing in the finite volume with periodic boundary conditions is explained in Figure 3.b.

It is now important to understand what happens if we deform the theory away from this integrable situation, moving into the fourth quadrant by means of the perturbation $g_{2} \int \varepsilon(x) \mathrm{d} x\left(g_{2}>0\right)$. The drastic effect of this perturbation is to lift the degeneracy of the three vacua: in the perturbed theory, the central vacuum $|0\rangle$ becomes the true ground state of the theory, whereas the other two vacua $| \pm\rangle$ become metastable ground states, separated from $|0\rangle$ by a gap

$$
\Delta E \sim g_{2}(\langle\Omega|\epsilon| \Omega\rangle-\langle 0|\epsilon| 0\rangle)
$$

where $|\Omega\rangle=| \pm\rangle$. As a consequence, we expect that the kinks of the unperturbed system will get confined as soon as we move away from $\eta_{+}=-\infty$ by switching on the coupling $g_{2}$ of the energy operator $\epsilon$. This can be directly seen by the FFPT, reported in Appendix C: the two-particle form factors on the kink states of the $\epsilon$ operator have in fact a pole at $\theta=\mathrm{i} \pi[23$. The linear confining potential between the constituents of the two pairs of neutral kink-antikink states $\left|K_{0-} K_{-0}\right\rangle$ and $\left|K_{0+} K_{+0}\right\rangle$ gives rise then to a dense sequence of bound states, with the number of bound states going to infinity as $g_{2} \rightarrow 0$ [7] (see Figure 5).

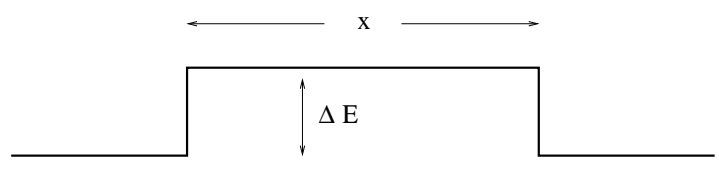

(a)

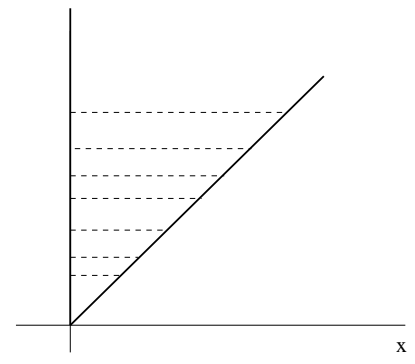

(b)

Figure 5. (a) Kink-antikink state at distance $x$, where $\Delta E$ is the gap of the unbalanced vacua. The kink and the antikink are subject to a linear confining potential (b), with slope given by $\Delta E$, and they give rise to an infinite sequence of bound states.

This analytic result is indeed confirmed by our numerical calculations. In particular, Figure 66 shows that the number of particles below the stability threshold increases to infinity as the first order transition line is approached (i.e. $\eta_{+} \rightarrow-\infty$ ). This scenario is similar to the one observed in the scaling region of the critical Ising model near the thermal axis once it is perturbed by a magnetic field [6] and it realizes the so-called McCoy-Wu scenario [22]. An important difference from the Ising model, however, is the $\mathbb{Z}_{2}$ symmetry of our model. At $\eta_{+}=-\infty$, the two-kink states $\left|K_{0-} K_{-0}\right\rangle$ and $\left|K_{0+} K_{+0}\right\rangle$ (the rapidities of the kinks are suppressed) are mapped into each other by spin reversal, so they form degenerate pairs (although in finite volume this degeneracy is lifted). The two-kink states 
$\left|K_{-0} K_{0-}\right\rangle$ and $\left|K_{+0} K_{0+}\right\rangle$ also form similar degenerate pairs, and so do the two-kink states $\left|K_{-0} K_{0+}\right\rangle$ and $\left|K_{+0} K_{0-}\right\rangle$. The degeneracy is lifted as soon as the perturbation is switched on; the arising bound states are singlets with definite parity. As the kinks do not have bound states at $\eta_{+}=-\infty$, all the bound states in the neighbourhood $g_{2}>0$ of the transition point arise from the free two-kink configurations mentioned above. Since the $| \pm\rangle$ vacua are lifted, the original two-particle states $\left|K_{-0} K_{0-}\right\rangle,\left|K_{+0} K_{0+}\right\rangle,\left|K_{-0} K_{0+}\right\rangle$, and $\left|K_{+0} K_{0-}\right\rangle$ of the continuum disappear entirely from the spectrum. In their place, there is a sequence of bound states $\left|B_{n}^{ \pm}\right\rangle$, which arises from the even and odd superpositions of the $\left|K_{0-} K_{-0}\right\rangle$ and $\left|K_{0+} K_{+0}\right\rangle$ states:

$$
\left|B_{n}^{ \pm}\right\rangle \sim \frac{\left|K_{0-} K_{-0}\right\rangle \pm\left|K_{0+} K_{+0}\right\rangle}{\sqrt{2}} .
$$

The superscript in $\left|B_{n}^{ \pm}\right\rangle$denotes parity, the subscript numbers the elements of the tower of bound states. As we mentioned, $\left|B_{n}^{+}\right\rangle$and $\left|B_{n}^{-}\right\rangle$are not degenerate. Due to the exactness of the $\mathbb{Z}_{2}$ symmetry the vacuum is expected to be even, and then the first state above it, i.e. the lowest one-particle state, is expected to be odd, and generally the parity is expected to alternate in the sequence of one-particle states.

The numerical data that we obtained by varying $\eta_{+}$indeed confirm that in the high temperature phase the spin reversal symmetry is unbroken, there is a unique vacuum and the excitations are singlet scalar particles with alternating parity. The disappearance of the two-kink states $\left|K_{-0} K_{0-}\right\rangle,\left|K_{+0} K_{0+}\right\rangle,\left|K_{-0} K_{0+}\right\rangle$ and $\left|K_{+0} K_{0-}\right\rangle$ does not have a drastic effect on the numerical spectrum on the cylinder with periodic boundary conditions, since the $\left|K_{-0} K_{0+}\right\rangle$, and $\left|K_{+0} K_{0-}\right\rangle$ are filtered out and $\left|K_{-0} K_{0-}\right\rangle$ and $\left|K_{+0} K_{0+}\right\rangle$ are identified with $\left|K_{0-} K_{-0}\right\rangle$ and $\left|K_{0+} K_{+0}\right\rangle$ under these boundary conditions: what happens is that, energy lines that were previously asymptotic degenerate, now become separated by tiny gaps.

The doubly degenerate false vacuum can be seen in the $\eta_{+}<0$ domain in the TCSA spectra in the form of a linearly rising double line-like pattern (see Figure 9,a). The slope of the pattern, which equals to the gap $\Delta E$ between the false and true vacua, increases from 0 as $\eta_{+}$is increased, and finally the pattern disappears from the finite volume spectrum as $\eta_{+}$approaches $0 . \Delta E$ can be calculated by FFPT around the first order phase transition line. At $g_{4}=-1$ the exact result to first order in perturbation theory is

$$
\frac{\Delta E}{g_{2}}=0.3076 \ldots=2 \Lambda_{2} \times(2 \pi)^{\frac{1-\Delta_{2}-\Delta_{4}}{1-\Delta_{4}}} \lambda_{4}^{\frac{\Delta_{2}}{1-\Delta_{4}}} \lambda_{2}
$$

where

$$
\Lambda_{2}=2.668319 \ldots
$$

is the exact value of the vacuum expectation values of $\varepsilon$ given in [25, 26], and the factor next to $2 \Lambda_{2}$ arises from various normalization factors used in the present paper and in [25, 26] (see Appendix B for more details on the calculation of $\Delta E$ ). On the other hand, by TCSA we obtain

$$
\frac{\Delta E}{g_{2}}=0.29
$$


The particle spectrum of the TIM with spin reversal symmetric perturbations

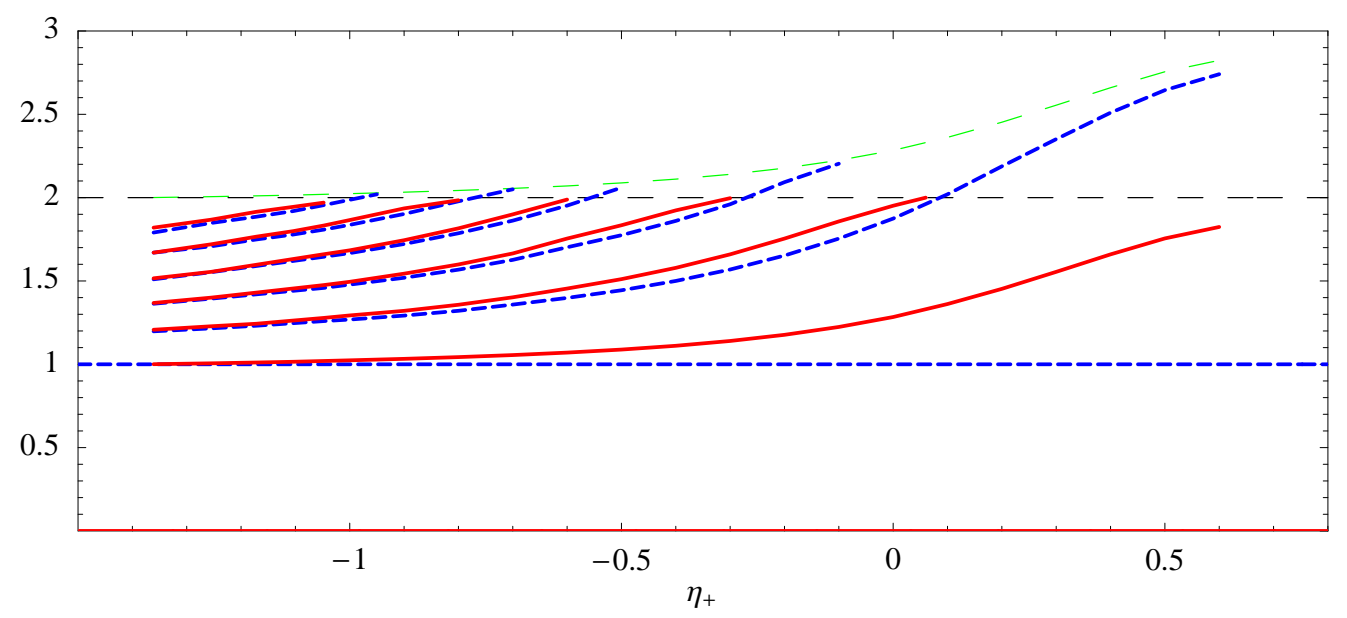

Figure 6. The particle mass ratios $m_{i} / m_{1}$ as functions of $\eta_{+}$in the high temperature phase in the range $-1.361 \leq \eta_{+} \leq 0.6$, obtained by TCSA. Even states are shown in red, odd states in blue and with dashed line. The thresholds $2 m_{1}$ and $m_{1}+m_{2}$ for even and odd particles are drawn with dashed thin black and green lines.

which is in reasonable agreement with the exact value.

Figure [6] shows the numerically calculated mass ratios $m_{i} / m_{1}\left(m_{1}\right.$ being the mass of the lightest particle) as functions of $\eta_{+}$. Truncation errors tend to increase for large absolute values of $\eta_{+}$, therefore the figure shows only a limited range around $\eta_{+}=0$. The masses $m_{1}$ and $m_{2}$ of the two lightest particles are also shown in Figure 7 . The stability threshold, taking into account spin reversal symmetry, is $2 m_{1}$ for even particles and $m_{1}+m_{2}$ for odd particles (if there are no even particles, then the threshold is $3 m_{1}$ ).

Hence, increasing $\eta_{+}$, the number of stable excitations decreases. Around $\eta_{+}=0$, i.e. near to the positive horizontal axis, there are four stable excitations. However, precisely at $\eta_{+}=0$, the theory acquires three more stable particles, all of them above threshold. In order to understand the nature of the spectrum near to $\eta_{+}=0$, it is obviously more convenient to switch to the integrable formulation of the theory along the positive horizontal axis.

\subsection{The $E_{7}$-related line and FFPT}

As we mentioned in the introduction, the $\eta=0$ line corresponds to an integrable model related to the Toda field theory based on the exceptional Lie algebra $E_{7}$. In the high temperature phase, this integrable model has a spectrum composed of seven scalar particles $A_{i}, i=1 \ldots 7$. Their masses $m_{i}, i=1 \ldots 7$ (see Table 1) and S-matrix amplitudes are known exactly [13, 14].

Note that $A_{1}, A_{3}$ and $A_{6}$ are odd under spin reversal symmetry while the other particles are even. Only the first four particles have a mass below the stability thresholds $2 m_{1}$ and $m_{1}+m_{2}$. The higher three particles are nevertheless stable along this axis due to the integrability of the theory. 
The particle spectrum of the TIM with spin reversal symmetric perturbations

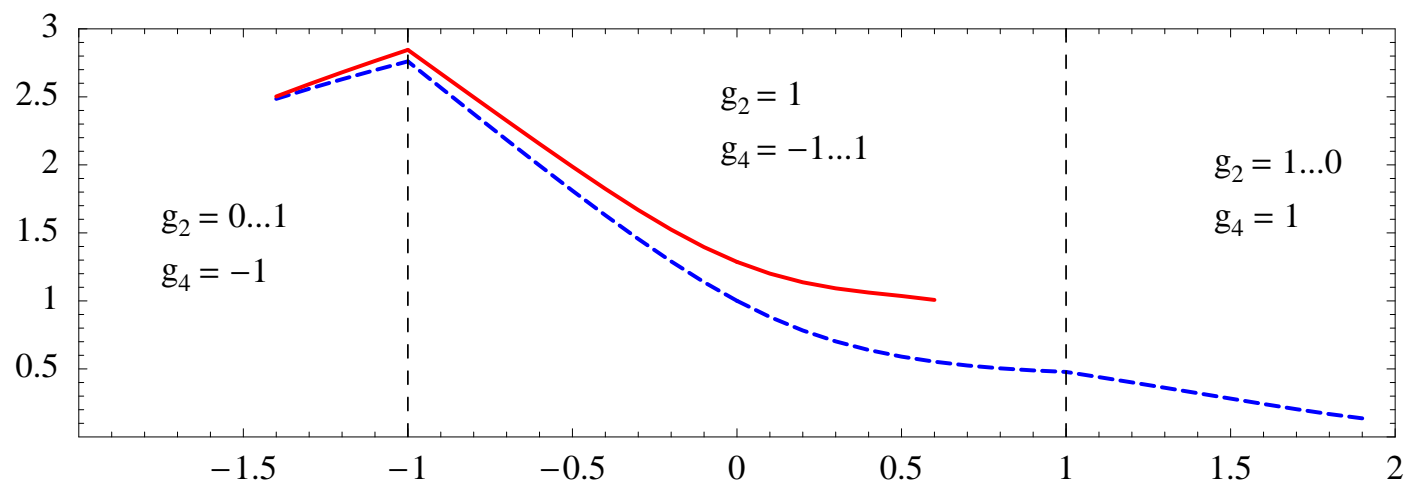

Figure 7. The particle masses $m_{1}$ (in red) and $m_{2}$ (in blue and with dashed line) obtained by TCSA along the square shown in Figure [ The section $0<g_{2}<0.6$, $g_{4}=-1$ is not shown.

Table 1. Masses and spin reversal parities of the particles on the $E_{7}$-related axis in the high temperature phase.

\begin{tabular}{|l|l|c|}
\hline mass & $m_{i} / m_{1}$ & $\mathbb{Z}_{2}$ parity \\
\hline$m_{1}$ & 1 & - \\
$m_{2}=2 m_{1} \cos (5 \pi / 18)$ & 1.28557 & + \\
$m_{3}=2 m_{1} \cos (2 \pi / 18)$ & 1.87938 & - \\
$m_{4}=2 m_{1} \cos (\pi / 18)$ & 1.96961 & + \\
$m_{5}=4 m_{1} \cos (\pi / 18) \cos (5 \pi / 18)$ & 2.53208 & + \\
$m_{6}=4 m_{1} \cos (4 \pi / 18) \cos (2 \pi / 18)$ & 2.87938 & - \\
$m_{7}=4 m_{1} \cos (\pi / 18) \cos (2 \pi / 18)$ & 3.70166 & + \\
\hline
\end{tabular}

\subsection{Decay processes}

Moving away from the axis $\eta_{+}=0$, the expectation is that there exists a range of values of $\eta_{+}$where the four lowest particles are still stable, whereas the higher three particles decay in all possible channels compatible with the symmetry of the perturbation. The perturbation that breaks the integrability of the theory at $\eta_{+}=0$ and plays the role of the operator $\Upsilon(x)$ in eqn (3.1) is the field $t$, which is even under the $\mathbb{Z}_{2}$ spin reversal symmetry. This implies certain selection rules in the decay processes. So, for instance, the particle $A_{5}$ can only decay in the channel

$$
A_{5} \rightarrow A_{1} A_{1}
$$

even though the decay process $A_{5} \rightarrow A_{1} A_{2}$ would be permitted by kinematics. Similarly, it is easy to check that the other possible decay processes compatible with the selection rule are

$$
\begin{aligned}
& A_{6} \rightarrow A_{1} A_{2} \\
& A_{7} \rightarrow A_{1} A_{1} \\
& A_{7} \rightarrow A_{2} A_{2} \\
& A_{7} \rightarrow A_{1} A_{3}
\end{aligned}
$$


The particle spectrum of the TIM with spin reversal symmetric perturbations

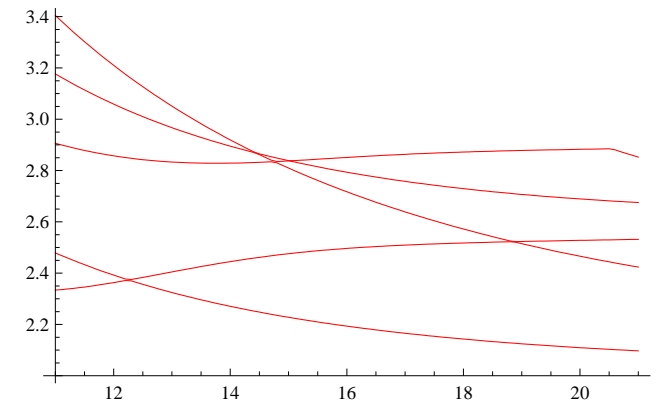

(a)

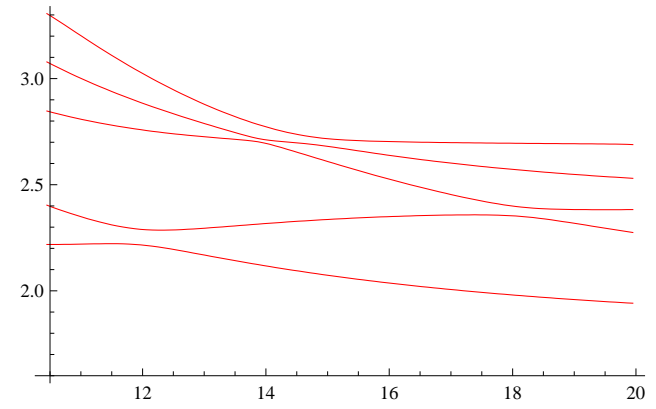

(b)

Figure 8. Signature of the appearance of a resonance out of the integrability in the finite volume spectrum. (a) Integrable point. (b) Perturbation switched on. These are the plots of the energy levels versus $m R$, where $m$ is the mass gap.

$$
\begin{aligned}
& A_{7} \rightarrow A_{2} A_{4} \\
& A_{7} \rightarrow A_{1} A_{1} A_{2} .
\end{aligned}
$$

The decay processes have a fingerprint in the finite volume spectra, namely the repulsion of the energy lines [6]. Observe, for instance, Figure 8,a: this refers to the lowest energy difference lines above the two-particle threshold (in the even sector only) on the integrable $E_{7}$ axis. One can see that there are several level crossings; in particular there is one around $m R \sim 12$ between the first and the second lines. The first line corresponds to the threshold state $\left|A_{1} A_{1}\right\rangle$, whereas the second line corresponds to the particle $\left|A_{5}\right\rangle$. When we switch on the $g_{4}$ perturbation, the crossing disappears, as it can clearly be seen in Figure 8. b. In particular, the repulsion of the first and second lines at $m R \sim 12$ corresponds to the decay $A_{5} \rightarrow A_{1} A_{1}$.

In principle, the widths $\Gamma_{i j}^{k}$ of the decays $A_{k} \rightarrow A_{i} A_{j}$ can be determined from the repulsion of energy lines by a method proposed in [28]. In this approach, which was called Breit-Wigner method in [28], $\Gamma$ is related to the two-particle phase shifts of the various two-particle levels $\left|A_{i} A_{j}\right\rangle$. In more detail, in order to measure $\Gamma$ one can use the formula

$$
\Delta \delta=\min \delta_{1}(E)-\max \delta_{2}(E)=4 \sqrt{-\beta \Gamma},
$$

where

$$
\beta=\left.\frac{\mathrm{d} \delta_{0}}{\mathrm{~d} E}\right|_{E=E_{k}}
$$

should be negative, $E_{k}$ is the center of the resonance and $\delta_{0}(E)$ is defined by the integrable S-matrix as

$$
\delta_{0}(E)=-\mathrm{i} \ln S(E) .
$$

For the sake of clarity, we have suppressed the labels $i, j, k$ relative to the particles. The symbols $\delta_{1}(E), \delta_{2}(E)$ in (4.8) refer to the phase shifts of two neighbouring two-particle levels $\left|A_{i} A_{j}\right\rangle$ and are given by

$$
\delta_{a}(E)=-R(E) p_{a}(E), \quad a=1,2,
$$


where $R$ is the circumference of the cylinder and $p_{a}(E)$ is the momentum, defined by the equation

$$
E=\sqrt{p_{a}^{2}+m_{i}^{2}}+\sqrt{p_{a}^{2}+m_{j}^{2}} .
$$

Hence, from the numerical determination of the energy levels we can measure $E(R)$ as a function of $R$; from this measurement, using (4.11), we can extract $\delta_{1}(E)$ and $\delta_{2}(E)$, and then (4.8) provides $\Gamma$.

Using the above consideration we have studied the decay processes

$$
\begin{aligned}
& A_{5} \rightarrow A_{1} A_{1} \\
& A_{6} \rightarrow A_{1} A_{2}
\end{aligned}
$$

at $g_{2}=1$ and small $g_{4}$, finding the following numerical values for the decay rates

$$
\Gamma_{11}^{5} \sim 2 \cdot g_{4}^{2}, \quad \Gamma_{12}^{6} \sim 0.2 \cdot g_{4}^{2},
$$

and therefore the universal ratio

$$
\frac{\Gamma_{12}^{6}}{\Gamma_{11}^{5}} \sim 0.1
$$

This result shows that the decay of $A_{6}$ is considerably slower than the decay of $A_{5}$. Although the kinematical phase space factor $\Phi_{m_{i}, m_{j}}^{M}$ generally tends to suppress the decay of heavier particles in $1+1$ dimensions [29], in the present case this effect is not sufficient, however, to justify the result above: in fact, the phase space estimate of the universal ratio (4.14) gives in this case

$$
\frac{\Gamma_{12}^{6}}{\Gamma_{11}^{5}} \sim \frac{\Phi_{12}^{6}}{\Phi_{11}^{5}}=\sqrt{\frac{m_{5}^{2}\left(m_{5}^{2}-4 m_{1}^{2}\right)}{\left(m_{6}^{2}-\left(m_{1}-m_{2}\right)^{2}\right)\left(m_{6}^{2}-\left(m_{1}+m_{2}\right)^{2}\right)}}=0.783 \ldots .
$$

Therefore the longer lifetime of $A_{6}$ has to be attributed to the dynamics of the model. We plan to analyze this aspect in a future publication.

\subsection{Mass corrections}

Coming back to the stable particles of the spectrum, it is relatively easy to compute the correction to the masses of the two lowest particles once we move away from the $E_{7}$ integrable axis. This can be done by combining the FFPT and the TCSA, together with a cross-checking between the two methods. The first order mass correction of the particle $A_{i}$ moving away from the $\eta_{+}=0$ axis is given by

$$
\delta m_{i}=\frac{g_{4}}{m_{i}} 2 \pi \lambda_{4} F_{i i}^{t}(\mathrm{i} \pi)
$$

where $F_{i i}^{t}\left(\theta_{1}-\theta_{2}\right)=\left\langle 0|t(0)| A_{i}\left(\theta_{1}\right) A_{i}\left(\theta_{2}\right)\right\rangle$ is the two-particle form factor of $t$. As a matter of fact, $F_{11}^{t}(\mathrm{i} \pi), F_{22}^{t}(\mathrm{i} \pi), F_{33}^{t}(\mathrm{i} \pi)$ and $F_{44}^{t}(\mathrm{i} \pi)$ can be directly extracted by TCSA (see Appendix B and [24, 26]). At $g_{2}=1$ we obtained

$$
\begin{array}{ll}
F_{11}^{t}(\mathrm{i} \pi)=-\frac{1.28}{2 \pi \lambda_{4}}, & F_{22}^{t}(\mathrm{i} \pi)=-\frac{1.25}{2 \pi \lambda_{4}}, \\
F_{33}^{t}(\mathrm{i} \pi)=-\frac{2.03}{2 \pi \lambda_{4}}, & F_{44}^{t}(\mathrm{i} \pi)=-\frac{3.41}{2 \pi \lambda_{4}} .
\end{array}
$$


Similarly, from TCSA we can also compute the absolute value of the one-particle form factors $F_{2}^{t}=\left\langle 0|t(0)| A_{2}(\theta)\right\rangle$ and $F_{4}^{t}=\left\langle 0|t(0)| A_{4}(\theta)\right\rangle$. The result is

$$
\left|F_{2}^{t}\right|=\frac{0.35}{2 \pi \lambda_{4}}, \quad\left|F_{4}^{t}\right|=\frac{0.26}{2 \pi \lambda_{4}} .
$$

As shown in the Appendix A, there is an exact relation between the two-particle form factors $F_{11}^{t}(i \pi)$ and $F_{22}^{t}(i \pi)$ and the one-particle form factors $F_{2}^{t}$ and $F_{4}^{t}$, expressed by

$$
\begin{aligned}
& F_{11}^{t}(\mathrm{i} \pi)=11.397 \cdot F_{2}^{t}+10.3632 \cdot F_{4}^{t} \\
& F_{22}^{t}(\mathrm{i} \pi)=23.1056 \cdot F_{2}^{t}+26.2404 \cdot F_{4}^{t} .
\end{aligned}
$$

If we assume that $F_{2}^{t}<0$ and $F_{4}^{t}>0$, then (4.19), (4.20) and (4.21) yield

$$
F_{11}^{t}(\mathrm{i} \pi)=\frac{-1.29}{2 \pi \lambda_{4}}, \quad F_{22}^{t}(\mathrm{i} \pi)=\frac{-1.26}{2 \pi \lambda_{4}},
$$

which is in satisfactory agreement with (4.17).

With the above values, we can extract the universal ratios

$$
\begin{aligned}
& \delta m_{2} / \delta m_{1}=0.76, \\
& \delta m_{3} / \delta m_{1}=0.84, \\
& \delta m_{4} / \delta m_{1}=1.35 .
\end{aligned}
$$

In order to get another universal ratio, let us look at the first order correction to the vacuum energy density $\epsilon_{v a c}$. By TCSA (see Appendix B) we obtain

$$
\frac{\delta \epsilon_{v a c}}{g_{4}}=0.11
$$

On the other hand, the exact value of this quantity is

$$
\frac{\delta \epsilon_{v a c}}{g_{4}}=0.1130 \ldots=\Lambda_{4} \times(2 \pi)^{\frac{1-\Delta_{2}-\Delta_{4}}{1-\Delta_{2}}} \lambda_{2}^{\frac{\Delta_{4}}{1-\Delta_{2}}} \lambda_{4}
$$

which is in good agreement with the above numerical result. $\Lambda_{4}=3.70708 \ldots$ is the vacuum expectation value of $t$ calculated in [25, 26] (the factor next to $\Lambda_{4}$ arises from various normalization factors used in the present paper and in [25, 26]). This allows us also to extract the universal ratio

$$
\frac{\delta \epsilon_{v a c}}{m_{1} \delta m_{1}}=-0.086
$$

\subsection{The first quadrant}

After the analysis in the previous section we can now proceed to investigate the evolution of the spectrum in the first quadrant. First of all, note that the corrections to the two lowest masses have the same sign, which depends on the sign of $g_{4}$. We can use the above results for $\delta m_{1}$ and $\delta m_{2}$ to estimate the value of $\eta_{+}^{(2)}$ where the mass $m_{2}\left(\eta_{+}\right)$of the second particle reaches the threshold $2 m_{1}\left(\eta_{+}\right)$of the lowest particle. This happens for a value of $\eta_{+}>0$ of the first quadrant, given by $\eta_{+}^{(2)} \simeq 1$ : although this is higher than the value $\eta_{+}^{(2)} \simeq 0.7$ extracted from the numerical data shown in Figure 6 , it is nevertheless 
a reasonable estimation of this quantity since the theoretical result was based on just first-order perturbation theory.

Besides the three higher particles $A_{5}, A_{6}, A_{7}$ that were stable only at $\eta_{+}=0$ and decay as soon as we move away from the horizontal axis, the TCSA analysis shows that the same pattern as was seen in the fourth quadrant also persists in the first quadrant: namely, increasing the value of $\eta_{+}$, the number of stable particles continues to decrease. The first particle that disappears into the threshold $2 m_{1}\left(\eta_{+}\right)$is $A_{4}$ at the critical value $\eta_{+}^{(4)}$, which can be estimated from first-order perturbation theory to be $\eta_{+}^{(4)} \simeq 0.04$. This is followed by $A_{3}$ that disappears into its lowest threshold, given by $\left(m_{1}\left(\eta_{+}\right)+m_{2}\left(\eta_{+}\right)\right)$, at the critical value $\eta_{+}^{(3)}$, for which perturbation theory gives $\eta_{+}^{(3)} \simeq 0.62$. These theoretical results for $\eta_{+}^{(3)}$ and $\eta_{+}^{(4)}$ are in reasonably good agreement with the numerical data (Figure 6). An example of the finite volume spectra calculated numerically is shown in Figure 10, a at $\eta_{+}=0.3$, where the theory contains 3 stable particles.

As just described, moving toward the positive vertical axis, there is a depletion of the number of stable particles, until there remains only one in the neighbourhood of $\eta_{+}=+\infty$. At the same time, notice that increasing $\eta_{+}$, the value of this lowest mass becomes also smaller 4 . The mass gap of the theory finally vanishes when $\eta_{+}=+\infty$, i.e. when we have reached the vertical axis.

During this evolution, however, there has also been a qualitative change of the spectrum: in fact, reaching the positive vertical axis the lowest excitation has turned into a fermion. This becomes evident by looking directly at the nature of the theory at $\eta_{+}=+\infty$ (notice that the positive vertical axis also corresponds to $\eta_{-}=+\infty$ ).

\subsection{The spontaneously SUSY breaking axis}

The analytic control of the theory in the vicinity of $\eta_{ \pm}=+\infty$ is provided by the integrability of the model along the vertical axis, where the system is also supersymmetric. Along the positive vertical axis the supersymmetry is however spontaneously broken: the low-energy excitations are given in this case by the massless right and left mover Majorana fermions, which play the role of goldstino [10, 11, 12]. The factorized scattering theory was proposed in [11] and the basic form factors were calculated in [21]. The massless Majorana fermions are nothing else than those of the Ising model, connected to the TIM by the massless renormalization group flow that occurs along this line.

Breaking the integrability of the $\eta_{ \pm}=\infty$ model by means of the operator $\Upsilon=\varepsilon$, the left and right moving excitations become adiabatically massive, as it can be directly observed in the TCSA data (see Figure 7). To compute the mass $m$ of the fermion generated by the perturbation, we need to employ in this case the massless FFPT [8]: at the lowest order, we have

$$
m \simeq g_{2} \lim _{\theta_{R L} \rightarrow-\infty} F_{R L}^{\varepsilon}\left(\mathrm{i} \pi+\theta_{R L}\right)
$$

4 This behavior is in agreement with the first-order correction (4.16) to $m_{1}$, that is negative for $g_{4}>0$. 
where the two-particle (right-left) form factor of the $\varepsilon$ operator is given by [21]

$$
F_{R L}^{\varepsilon}(\theta)=\exp \left(\frac{\theta}{4}-\int_{0}^{\infty} \frac{\mathrm{d} t}{t} \frac{\sin ^{2}\left(\frac{(\mathrm{i} \pi-\theta) t}{2 \pi}\right)}{\sinh t \cosh \frac{t}{2}}\right) .
$$

Using (4.27) and (4.28), one can easily check that $m$ is a finite quantity, positive for $g_{2}>0$ and negative for $g_{2}<0$. Note that, for a Majorana fermion, a negative value of the mass signals that we are in a low temperature phase, i.e. that the theory has two degenerate vacua. This is the topic of the next section.

\section{The spectrum in the low temperature phase}

The low temperature phase is composed of the second and the third quadrants. This phase is described by the variable $\eta_{-} \in(-\infty,+\infty): \eta_{-}=\infty$ corresponds to the positive vertical axis, $\eta_{-}=0$ to the negative horizontal axis and $\eta_{-}=-\infty$ to the negative vertical axis.

In this phase the model presents generically two degenerate ground states $|-\rangle$ and $|+\rangle$, which are mapped into each other by the spin reversal symmetry operation $Q:|+\rangle=Q|-\rangle$. An important feature that is worth stressing is the following: no matter how we vary the coupling constants in this parameter region, the two vacua $|-\rangle$ and $|+\rangle$ always remain degenerate, the only changes being in the shape and the height of the barrier between them. Hence, in this model the kink states that interpolate between the two vacua are always stable excitations. As we will comment below, this can be explicitly checked and confirmed by a FFPT computation at $\eta_{-}=0$, where the theory has the $E_{7}$ structure.

The only points where one has to be careful are the two limiting cases $\eta_{-}=\infty$ and $\eta_{-}=-\infty$. In the former case, the barrier between the two vacua disappears and, as we saw at the end of Section 4.5, the kinks become massless. In the latter case, the two vacua $|-\rangle$ and $|+\rangle$ and the false vacuum $|0\rangle$ that emerges between them in the region $\eta_{-}<0$ become degenerate precisely at $\eta_{-}=-\infty$. Hence, at this point, the original kinks break into a new set of smaller kinks, i.e. those relative to the three vacua of the first order phase transition of the TIM.

To obtain the spectrum of the theory in the low temperature phase we can take advantage of the duality of the model. At a formal level, note that the masses at a given point $\left(g_{2}, g_{4}\right)$ in the low temperature phase are the same as the masses in the dual point $\left(-g_{2}, g_{4}\right)$ in the high temperature phase. This result can be obtained as follows: since $\varepsilon$ is odd and $t$ is even under the duality transformation $D$ :

$$
\begin{aligned}
& D^{-1} \varepsilon D=-\varepsilon, \\
& D^{-1} t D=t,
\end{aligned}
$$

for the Hamiltonian we have

$$
D^{-1} H\left(g_{2}, g_{4}\right) D=H\left(-g_{2}, g_{4}\right) \text {. }
$$

However, to interpret correctly the TCSA data, one needs to take into account the existence of kink states and the periodic boundary conditions imposed to the system. 
Note that $D$ maps the even sector of the Hilbert space onto itself, so in the even sector $H\left(g_{2}, g_{4}\right)$ has the same spectrum as $H\left(-g_{2}, g_{4}\right)$. Concerning the odd sector of the spectrum, the particles with odd parity in the high temperature phase become instead kinks in the low temperature phase, i.e. topologically charged states that interpolate between the two vacua. However, the periodic boundary conditions that we have imposed on the TCSA filter only topologically neutral states. Hence, by switching $g_{2} \rightarrow-g_{2}$, we expect that the net result in the numerical outcomes will be the disappearing of the energy levels corresponding to the odd particles in the high temperature phase: in the low temperature phase these lines should become exponentially degenerate with the energy lines of the even levels. In other words, in the low temperature phase the TCSA data should show doubly degenerate lines and each doublet should have an even and odd member. This is indeed the case, as clearly shown in Figures 9 and 10, where we present the spectrum of the theory at given values $\left(g_{2}, g_{4}\right)$ and $\left(-g_{2}, g_{4}\right)$. This feature is nothing but a finite volume manifestation of the spontaneous breaking of the $\mathbb{Z}_{2}$ spin reversal symmetry: the two vacua (and all the excitations above them) have in fact an exponential splitting of their energy for the tunneling phenomenon that occurs at finite volume.

Summarizing the considerations above, in the low temperature phase and at infinite volume the model has two ground states $|+\rangle,|-\rangle$, each ground state has a sequence $\left|C_{n}\right\rangle_{ \pm}$of topologically neutral particles above it, and there is also a sequence of kinks and antikinks $\left|\mathcal{D}_{+-}^{m}\right\rangle,\left|\mathcal{D}_{-+}^{m}\right\rangle$ which interpolate between the vacuas. Under spin reversal symmetry the ground states, particles and kinks have the following property:

$$
\begin{aligned}
& | \pm\rangle=Q|\mp\rangle ; \\
& \left|C_{n}\right\rangle_{ \pm}=Q\left|C_{n}\right\rangle_{\mp} ; \\
& \left|\mathcal{D}_{+-}^{n}\right\rangle=Q\left|\mathcal{D}_{-+}^{n}\right\rangle .
\end{aligned}
$$

The masses of the neutral states at a given point of the plane of the coupling constants are the same as the masses of the even particles in the high temperature phase at the dual point, whereas the masses of the kinks are the same as the masses of the odd particles in the high temperature phase. In finite volume with periodic boundary conditions, however, we do not see individual kinks or other topologically charged configurations. We do see the vacua, the neutral particles and only neutral configurations of two or other even number of free kinks, which, moreover, get mixed in finite volume into even and odd eigenstates of the spin reversal operator and acquire an exponential splitting due to tunneling effects. With periodic boundary conditions there are also identifications between certain configurations, similarly as in the first order transition point (see Figure 3).

After the discussion above we can proceed to describe the spectrum in the second and third quadrants in detail.

5 The indices $n$ and $m$ number the members of the sequences, while + and - refer to the vacua between which the kinks interpolate or which support the neutral states. 


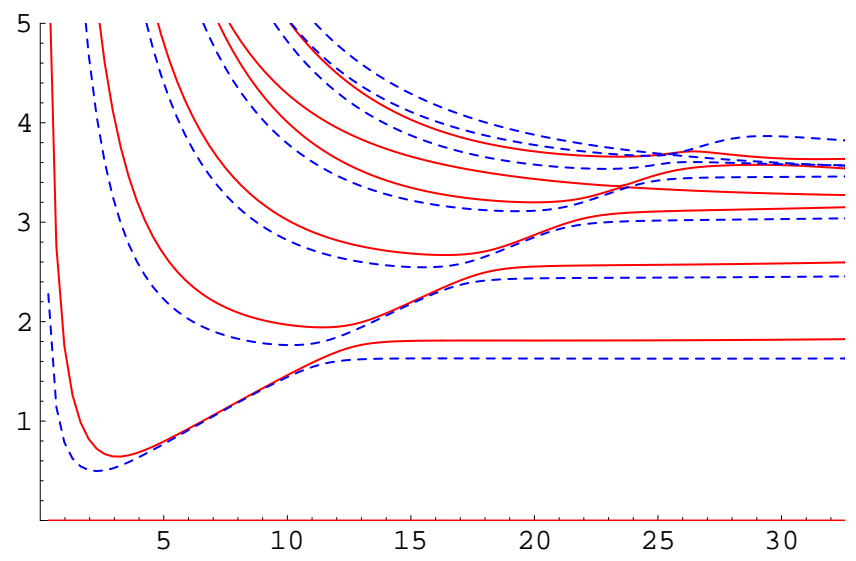

(a) $g_{2}=1, g_{4}=-0.4$

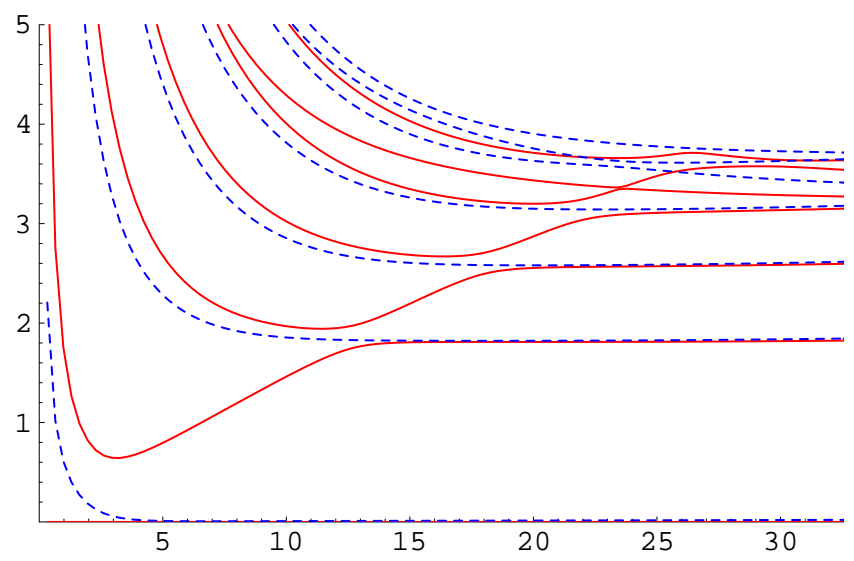

(b) $g_{2}=-1, g_{4}=-0.4$

Figure 9. The first 14 energy level differences $E_{i}-E_{0}, i=0 \ldots 13$, as functions of $m R$ in the (a) 4th and (b) 3rd quadrants at the dual values of the couplings constants. Even levels are in red, odd levels are in blue with dashed line. $m$ denotes the mass gap.

\subsection{The third quadrant}

At $\eta_{-}=-\infty$ the model is at the first order phase transition point, which was described in Section 4.1. As we move into the third quadrant by switching on the perturbation $g_{2} \int \varepsilon(x) \mathrm{d} x\left(g_{2}<0\right)$, the degeneracy of the three vacua is lifted. In contrast with the fourth quadrant, the central vacuum $|0\rangle$ now becomes a metastable ground state, and the two other vacua $| \pm\rangle$ remain true ground states. The gap $\Delta E$ separating $|0\rangle$ from $| \pm\rangle$ at a given point is equal to the gap at the dual point in the fourth quadrant. The expected consequence of the lifting of the degeneracy of the vacua is again the confinement of the kinks of the unperturbed system, which is confirmed by the same FFPT argument as for the fourth quadrant. The linear confining potential between the kinks gives rise to a dense sequence of bound states. As in the fourth quadrant, the number of these bound states goes to infinity as $g_{2} \rightarrow 0$. In the third quadrant, however, the two-kink states $\left|K_{0-} K_{-0}\right\rangle$ 


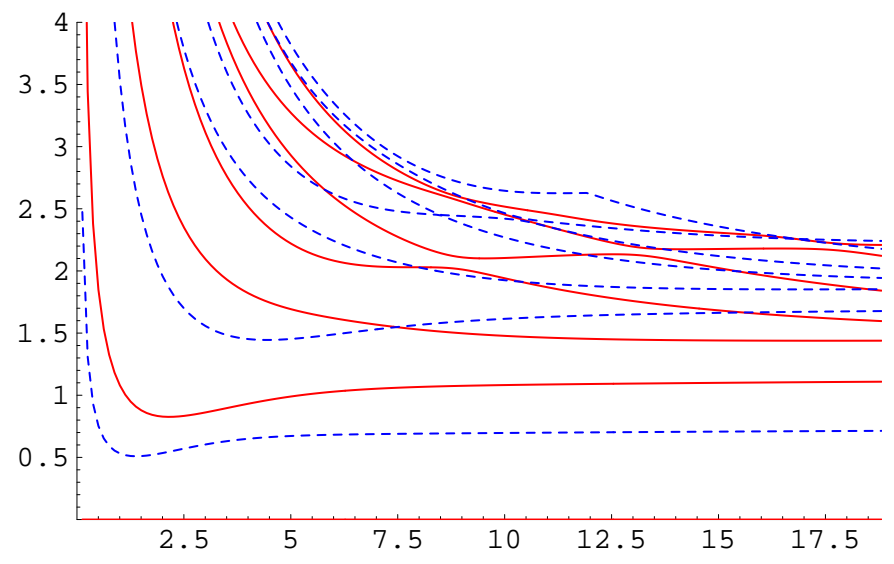

(a) $g_{2}=1, g_{4}=0.3$

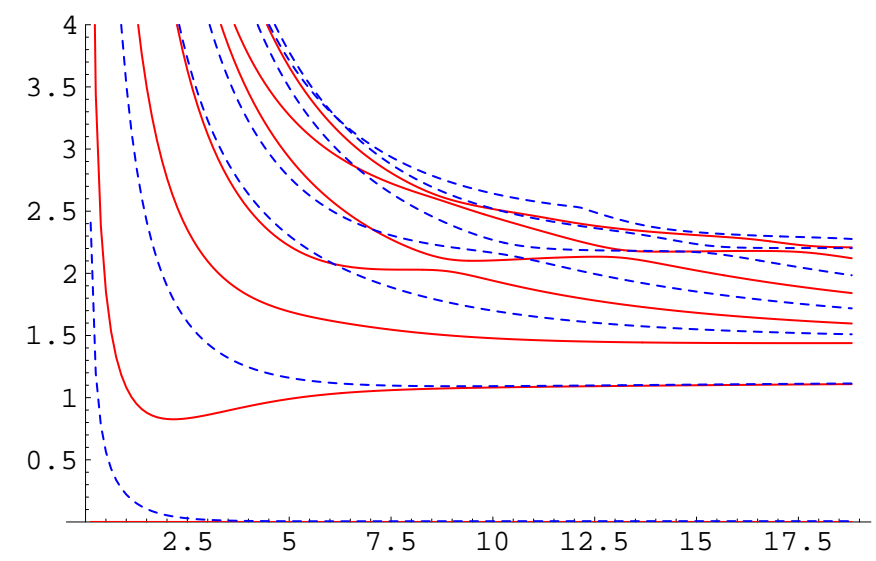

(b) $g_{2}=-1, g_{4}=0.3$

Figure 10. The first 14 energy level differences $E_{i}-E_{0}, i=0 \ldots 13$, as functions of $m R$ in the (a) 1st and (b) 2nd quadrants at the dual values of the coupling constants. Even levels are in red, odd levels are in blue with dashed line. $m$ denotes the mass gap.

and $\left|K_{0+} K_{+0}\right\rangle$ are those which disappear from the spectrum, and the bound states arise from the other two-kink states $\left|K_{-0} K_{0+}\right\rangle,\left|K_{+0} K_{0-}\right\rangle,\left|K_{-0} K_{0-}\right\rangle$ and $\left|K_{+0} K_{0+}\right\rangle$. Some of the bound states above are topologically charged, i.e. they are kinks, while others are neutral. The neutral particles arise from the neutral two-kink configurations:

$$
\left|C^{n}\right\rangle_{+} \sim\left|K_{+0} K_{0+}\right\rangle, \quad\left|C^{n}\right\rangle_{-} \sim\left|K_{-0} K_{0-}\right\rangle .
$$

It is important to note that $\left|C^{n}\right\rangle_{+}$and $\left|C^{n}\right\rangle_{-}$are degenerate for any given $n$, due to the spontaneously broken $\mathbb{Z}_{2}$ symmetry, although their degeneracy is exponentially lifted in finite volume. The topologically charged particles arise from topologically charged two-kink states:

$$
\left|\mathcal{D}_{-+}^{n}\right\rangle \sim\left|K_{-0} K_{0+}\right\rangle, \quad\left|\mathcal{D}_{-+}^{n}\right\rangle \sim\left|K_{-0} K_{0+}\right\rangle,
$$

where the subscripts -+ and +- in $\left|\mathcal{D}_{-+}^{n}\right\rangle$ and $\left|\mathcal{D}_{+-}^{n}\right\rangle$ denote the vacua between which these kink configurations interpolate. $\left|\mathcal{D}_{-+}^{n}\right\rangle$ and $\left|\mathcal{D}_{+-}^{n}\right\rangle$ are mapped into each other by 
the spin reversal: $Q\left|\mathcal{D}_{-+}^{n}\right\rangle=\left|\mathcal{D}_{+-}^{n}\right\rangle$, therefore they have equal mass.

As we mentioned, under duality an even particle $\left|B_{n}^{+}\right\rangle$in the high temperature phase corresponds to the neutral particles $\left|C^{n}\right\rangle_{ \pm}$of the same mass in the low temperature phase, and an odd particle $\left|B_{n}^{-}\right\rangle$corresponds to a kink-antikink pair $\left|\mathcal{D}_{+-}^{n}\right\rangle,\left|\mathcal{D}_{-+}^{n}\right\rangle$ of the same mass.

The neutral particles $\left|C^{n}\right\rangle_{ \pm}$(more precisely, their even and odd superpositions) can indeed be seen in the TCSA spectra, whereas the kinks $\left|\mathcal{D}_{+-}^{n}\right\rangle$ and $\left|\mathcal{D}_{-+}^{n}\right\rangle$ are filtered out by the periodic boundary conditions. The neutral two-kink states constituted by $\left|\mathcal{D}_{+-}^{n}\right\rangle$ and $\left|\mathcal{D}_{-+}^{n}\right\rangle$ can nevertheless be observed in the numerical data. The disappearance of the original two-kink states $\left|K_{0-} K_{-0}\right\rangle$ and $\left|K_{0+} K_{+0}\right\rangle$ does not have drastic effect on the spectra with periodic boundary conditions for similar reasons as for the third quadrant.

The false vacuum with even parity can also be seen in the $\eta_{-}<0$ domain in the TCSA spectra in the form of a linearly rising line-like pattern (see Figure 9,b). The duality relates this metastable vacuum to the metastable vacua in the first quadrant.

\subsection{The $E_{7}$-related line}

The number of stable particles at any given point in the low temperature phase is equal to the number of particles in the dual point in the high temperature phase, and this number decreases as $\eta_{-}$is increased. The number of stable particles around $\eta_{-}=0$ is 4 , however at $\eta_{-}=0$, i.e. on the negative horizontal axis, 3 more particles become stable above threshold. These particles are related by duality to the three highest particles existing at $\eta_{+}=0$. In particular, there are two neutral particles $\left|C^{5}\right\rangle_{ \pm},\left|C^{7}\right\rangle_{ \pm}$of masses $m_{5}$ and $m_{7}$ (see Table 1) which correspond to the even particles $\left|A_{5}\right\rangle,\left|A_{7}\right\rangle$, and there is one kink-antikink pair $\left|\mathcal{D}_{+-}^{6}\right\rangle,\left|\mathcal{D}_{-+}^{n}\right\rangle$ of mass $m_{6}$ corresponding to the odd particle $\left|A_{6}\right\rangle$.

It was stressed in the introduction that an important property of the model is the presence of kinks in the low temperature phase which do not get confined under perturbations. At $\eta_{-}=0$ this non-confinement can be explicitly verified by FFPT. For this, one has to show that the form factors giving the first-order corrections to the masses of $\left|\mathcal{D}_{+-}^{i}\right\rangle$ and $\left|\mathcal{D}_{-+}^{i}\right\rangle$ are finite. This is indeed the case, since these form factors

$$
\begin{aligned}
& \left\langle-|t(0)| \mathcal{D}_{-+}^{i}(\mathrm{i} \pi) \mathcal{D}_{+-}^{i}(0)\right\rangle, \\
& \left\langle+|t(0)| \mathcal{D}_{+-}^{i}(\mathrm{i} \pi) \mathcal{D}_{-+}^{i}(0)\right\rangle,
\end{aligned}
$$

(where $i=1,3)$ are equal by duality to the form factors $\left\langle 0|t(0)| A_{i}(\mathrm{i} \pi) A_{i}(0)\right\rangle$, and the latter are finite since $t$ does not have non-trivial semi-local index with respect to the $\left|A_{i}\right\rangle$ particles, which are local excitations.

The particles $\left|C^{5}\right\rangle_{ \pm},\left|C^{7}\right\rangle_{ \pm},\left|\mathcal{D}_{+-}^{6}\right\rangle,\left|\mathcal{D}_{-+}^{6}\right\rangle$ are unstable near the $\eta_{-}=0$ axis, the allowed decay processes can be obtained from (4.6) and (4.7) by replacing $A_{i}$ by the appropriate dual particles. For example, (4.6) corresponds to

$$
C_{+}^{5} \rightarrow \mathcal{D}_{+-}^{1} \mathcal{D}_{-+}^{1}, \quad C_{-}^{5} \rightarrow \mathcal{D}_{-+}^{1} \mathcal{D}_{+-}^{1}
$$




\subsection{The second quadrant}

Similarly to the third quadrant, the particle spectrum in the second quadrant is related by duality to the spectrum in the first quadrant. Even particles in the first quadrant correspond to neutral particles, odd particles correspond to kinks. The number of particles

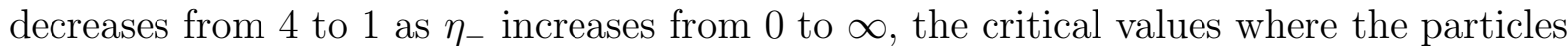
and kinks cross the threshold are the same as in the first quadrant, i.e. $\eta_{+}^{(4)}, \eta_{+}^{(3)}, \eta_{+}^{(2)}$. For $\eta_{-}>\eta_{+}^{(2)}$ there is only one kink-antikink pair in the spectrum. Similarly to the first quadrant, metastable vacua are not present. As $\eta_{-}$goes to infinity, the potential barrier between the two vacua $| \pm\rangle$ and the mass of the kink-antikink pair decrease until they finally vanish at the second-order transition point $\eta_{-}=\infty$. An example of the finite volume spectra calculated numerically is shown in Figure 10, b at $\eta_{-}=0.3$, which is dual to the point $\eta_{+}=0.3$ that the example Figure 10, a for the first quadrant shows.

\section{Conclusions}

In this paper we have studied the particle spectrum of the TIM with spin reversal symmetric perturbations. This is the simplest bosonic non-integrable field theory where kink excitations do not get confined by changing the coupling constants over a wide range of values. By varying the couplings, the model interpolates between a SUSY theory (either in its exact or in its spontaneouly broken phase) and a theory ruled by the exceptional algebra $E_{7}$, with a spectrum given by purely scalar particles (in the high temperature phase) and kinks and bound states thereof (in the low temperature phase).

Our results, obtained both by the FFPT and TCSA methods, are also in agreement with the Landau-Ginzburg picture of the TIM. We have found that the particle masses are the same at dual points in the high and low temperature phases, whereas the spin reversal symmetry properties and the topological charges of the particles are different in the two phases: even particles in the high temperature phase correspond to topologically neutral particles in the low temperature phase, odd particles correspond to (topologically charged) kink states. The number of stable particles tends to infinity in the vicinity of the first-order transition line, which is similar to the accumulation of particles found in the low temperature Ising model perturbed by a magnetic field [22]. However, in contrast with the Ising model, in TIM the parity is a good quantum number and the corresponding particles emerging from the kink-antikink threshold carry even or odd parity eigenvalues.

We have also computed the first-order corrections to the lowest four masses and the vacuum energy density at the $E_{7}$-related line, as well as the corrections to the energy densities of the three degenerate vacua at the first-order phase transition line. All these analytic results are in good agreement with the numerical estimates extracted by means of the TCSA.

Finally, we would like to mention that the three-frequency sine-Gordon model also has a tricritical point, the neighbourhood of which was studied by the TCSA in [30, 31]. In the light of the results of this paper, it would be interesting to investigate more thoroughly 
the first order transition in the three-frequency sine-Gordon model and, in particular, to study the evolution of the particle spectrum of this model. Moreover, the strategy adopted in the present paper can be also used to study the spectrum of higher multicritical theories, described by higher minimal models of conformal field theories perturbed by several operators.

\section{Acknowledgements}

We would like to thank Gesualdo Delfino and Robert Konik for useful discussions. 


\section{Appendix A}

\section{Form Factor Bootstrap}

In this appendix we explain the derivation of the relations (4.20) and (4.21) by the form factor approach. The two-particle form factor $F_{i i}^{t}(\theta)$ can be written as [7]

$$
F_{i i}^{t}(\theta)=Q_{i i}^{t}(\theta) \frac{F_{i i}^{\min }(\theta)}{D_{i i}(\theta)},
$$

where $F_{i i}^{\min }(\theta)$ and $D_{i i}(\theta)$ can be obtained by specializing the general formulas [7] to the present case:

$$
\begin{aligned}
& D_{11}(\theta)=P_{10}(\theta) P_{2}(\theta) \\
& D_{22}(\theta)=P_{12}(\theta) P_{8}(\theta) P_{2}(\theta) \\
& F_{11}^{\text {min }}(\theta)=-\mathrm{i} \sinh \frac{\theta}{2} g_{10}(\theta) g_{2}(\theta) \\
& F_{22}^{\text {min }}(\theta)=-\mathrm{i} \sinh \frac{\theta}{2} g_{12}(\theta) g_{8}(\theta) g_{2}(\theta),
\end{aligned}
$$

where

$$
\begin{aligned}
& P_{n}(\theta)=\frac{\cos \left(\frac{n}{18} \pi\right)-\cosh \theta}{2 \cos ^{2}\left(\frac{n}{18} \frac{\pi}{2}\right)} \\
& g_{n}(\theta)=\exp \left[2 \int_{0}^{\infty} \frac{\mathrm{d} x}{x} \frac{\cosh [(n / 18-1 / 2) x]}{\cosh x / 2 \sinh x} \sin ^{2}[(\mathrm{i} \pi-\theta) x / 2 \pi]\right] .
\end{aligned}
$$

The $Q_{i i}^{t}(\theta)$ are polynomials in $\cosh (\theta)$. An upper bound on the degree $d_{P}$ of these polynomials can be obtained from the following general formula for the asymptotic behaviour of form factors [7]:

$$
\lim _{\left|\theta_{i}\right| \rightarrow \infty} F_{a_{1}, \ldots, a_{n}}^{\varphi}\left(\theta_{1}, \ldots, \theta_{n}\right) \sim \mathrm{e}^{y_{\varphi}\left|\theta_{i}\right|}, \quad y_{\varphi} \leq \Delta_{\varphi}
$$

This relationship fixes $d_{P}=1$ for both $Q_{11}^{t}(\theta)$ and $Q_{22}^{t}(\theta)$ :

$$
Q_{i i}^{t}(\theta)=a_{i i}^{t}+b_{i i}^{t} \cosh (\theta), \quad i=1,2 .
$$

These coefficients $a_{i i}^{t}$ and $b_{i i}^{t}$ can be expressed in terms of the three-particle coupling constants $\Gamma_{11}^{2}, \Gamma_{11}^{4}, \Gamma_{22}^{2}, \Gamma_{22}^{4}$ and the one-particle form factors $F_{2}^{t}, F_{4}^{t}$ by means of the residue equations

$$
-\mathrm{i} \lim _{\theta \rightarrow \mathrm{i} u_{a b}^{c}}\left(\theta-\mathrm{i} u_{a b}^{c}\right) F_{a b}^{\varphi}(\theta)=\Gamma_{a b}^{c} F_{c}^{\varphi}
$$

for the fusions

$$
\begin{array}{ll}
A_{1} \times A_{1} \rightarrow A_{2}, & u_{11}^{2}=\frac{10}{18} \pi, \\
A_{1} \times A_{1} \rightarrow A_{4}, & u_{11}^{4}=\frac{2}{18} \pi, \\
A_{2} \times A_{2} \rightarrow A_{2}, & u_{22}^{2}=\frac{12}{18} \pi,
\end{array}
$$


The particle spectrum of the TIM with spin reversal symmetric perturbations

$$
A_{2} \times A_{2} \rightarrow A_{4}, \quad u_{22}^{4}=\frac{8}{18} \pi
$$

where $u_{a b}^{c}$ denotes the fusion angle. The expressions we found are the following:

$$
\begin{aligned}
& a_{11}^{t}=1.14107 \cdot \Gamma_{11}^{2} F_{2}^{t}-1.77654 \cdot \Gamma_{11}^{4} F_{4}^{t} \\
& b_{11}^{t}=-1.21431 \cdot \Gamma_{11}^{2} F_{2}^{t}-10.2307 \cdot \Gamma_{11}^{4} F_{4}^{t} \\
& a_{22}^{t}=0.306459 \cdot \Gamma_{22}^{2} F_{2}^{t}-1.37383 \cdot \Gamma_{22}^{4} F_{4}^{t} \\
& b_{22}^{t}=-1.76483 \cdot \Gamma_{22}^{2} F_{2}^{t}-2.74766 \cdot \Gamma_{22}^{4} F_{4}^{t} .
\end{aligned}
$$

In terms of these coefficients

$$
F_{i i}^{t}(\mathrm{i} \pi)=a_{i i}^{t}-b_{i i}^{t}, \quad i=1,2 .
$$

The squares of three-particle coupling constants can be obtained from the S-matrix elements

$$
\begin{aligned}
& S_{11}(\theta)=-f_{10}(\theta) f_{2}(\theta) \\
& S_{22}(\theta)=f_{12}(\theta) f_{8}(\theta) f_{2}(\theta),
\end{aligned}
$$

where

$$
f_{n}(\theta)=\frac{\tanh \frac{1}{2}\left(\theta+\mathrm{i} \pi \frac{n}{18}\right)}{\tanh \frac{1}{2}\left(\theta-\mathrm{i} \pi \frac{n}{18}\right)}
$$

by using the residue equation

$$
-\mathrm{i} \lim _{\theta \rightarrow \mathrm{i} u_{a b}^{c}}\left(\theta-\mathrm{i} u_{a b}^{c}\right) S_{a b}(\theta)=\left(\Gamma_{a b}^{c}\right)^{2}
$$

for the fusions (A.8)-(A.11). We obtained

$$
\begin{array}{ll}
\left(\Gamma_{11}^{2}\right)^{2}=4.838705173^{2} & \left(\Gamma_{11}^{4}\right)^{2}=1.225805260^{2} \\
\left(\Gamma_{22}^{2}\right)^{2}=11.15518618^{2} & \left(\Gamma_{22}^{4}\right)^{2}=19.10015279^{2} .
\end{array}
$$

Assuming $\Gamma_{i i}^{2}>0, \Gamma_{i i}^{4}>0$, these results can be substituted into (A.12)-(A.15), and then (A.16) takes the form of (4.20) and (4.21).

\section{Appendix B}

\section{Calculation of form factors by TCSA}

A one-particle form factor of a field $\varphi$ of conformal weights $(\Delta, \Delta)$ at infinite $R$ can be obtained as the limit of the finite $R$ form factor:

$$
F_{A}^{\varphi}=\langle 0|\varphi(0)| A\rangle=\lim _{R \rightarrow \infty} R^{-2 \Delta} \sqrt{R m}\langle 0|\varphi(0)| A\rangle_{R},
$$

where the subscript $R$ on the right hand side indicates that the matrix element should be calculated in the theory defined at size $R$ and $m$ is the mass of the particle $|A\rangle$. In this formula $|A\rangle$ is a zero-momentum state and it is assumed that $\langle 0 \mid 0\rangle_{R}=\langle A \mid A\rangle_{R}=1$. In the framework of the TCSA the absolute value of the matrix elements $\langle 0|\varphi(0)| A\rangle_{R}$ can be calculated. For a general matrix element the formula

$$
\frac{\langle A|\varphi(0)| B\rangle}{\sqrt{\langle A \mid A\rangle} \sqrt{\langle B \mid B\rangle}}=\frac{\sum_{a, b} A_{a}^{*} B_{b}\langle a|\varphi(0)| b\rangle}{\sqrt{\sum_{a, b} G_{a b} A_{a}^{*} A_{b}} \sqrt{\sum_{a, b} G_{a b} B_{a}^{*} B_{b}}}
$$


holds, where the subscript $R$ is suppressed, it is not assumed that the eigenvectors $|A\rangle$ and $|B\rangle$ are normalized to unity, $A_{a}$ and $B_{b}$ are expansion coefficients with respect to the conformal basis used in the TCSA: $|A\rangle=\sum_{a} A_{a}|a\rangle,|B\rangle=\sum_{b} B_{b}|b\rangle$, and $G_{a b}=\langle a \mid b\rangle$ is the inner product matrix of the conformal basis vectors. The expansion coefficients $A_{a}$ and $B_{b}$ are calculated numerically up to overall constant factors; they are provided by the routine that one uses for the diagonalization of the Hamiltonian operator.

A two-particle form factor $F_{A A}^{\varphi}(\theta)$ at $\theta=\mathrm{i} \pi$ at infinite $R$ can be obtained as the following limit of finite volume matrix elements:

$$
\begin{aligned}
& F_{A A}^{\varphi}(\mathrm{i} \pi)=\langle A(0)|\varphi(0)| A(0)\rangle \\
& \quad=\lim _{R \rightarrow \infty} R^{-2 \Delta} R m\left(\langle A|\varphi(0)| A\rangle_{R}-\langle 0|\varphi(0)| 0\rangle_{R}\right) .
\end{aligned}
$$

It is assumed that $A$ is a self-conjugate particle, and normalization conditions similar to those for (B.1) apply. In the framework of the TCSA the matrix elements $\langle A|\varphi(0)| A\rangle_{R}$ and $\langle 0|\varphi(0)| 0\rangle_{R}$ can be calculated in a similar way as the matrix element in (B.1), i.e. by the formula $(\underline{\mathrm{B} .2})$.

\section{Appendix C}

\section{Calculation of corrections to vacuum energy densities}

The first order correction (4.24) to the vacuum energy density $\epsilon_{v a c}=\lim _{R \rightarrow \infty} E_{\text {vac }}(R) / R$ at $g_{2}=1, g_{4}=0$ on the $E_{7}$-related axis was calculated by the following formula:

$$
\delta \epsilon_{v a c}=g_{4} \frac{\mathrm{d} \epsilon_{v a c}}{\mathrm{~d} g_{4}}\left(g_{4}=0\right)=\lim _{R \rightarrow \infty} 2 \pi g_{4} \lambda_{4} R^{-2 \Delta_{4}}\langle 0|t(0)| 0\rangle_{R} .
$$

The numerical calculation of the first order corrections to the energy densities of the $|+\rangle,|-\rangle,|0\rangle$ vacua existing at the first order phase transition point is slightly more complicated, since these vacua are degenerate in infinite volume $(R=\infty)$. Because of this degeneracy, one has to use, in general, degenerate perturbation theory, i.e. one obtains the first-order corrections by calculating the eigenvalues of the 3 by 3 matrix constituted by the 9 matrix elements $\left\langle+,-, 0\left|H_{p}\right|+,-, 0\right\rangle$ of the perturbing operator $H_{p}$ between the three vacua. Those combinations of the vacua which evolve into the split vacua existing at nonzero perturbation are given by the eigenvectors of this matrix.

More precisely, in our case the first order corrections to the energy densities of the vacua are given by the eigenvalues of the matrix

$$
M_{i j}=\lim _{R \rightarrow \infty} 2 \pi g_{2} \lambda_{2} R^{-2 \Delta_{2}}\left\langle U_{i}|\varepsilon(0)| U_{j}\right\rangle_{R}
$$

where $i, j=1,2,3, g_{4}$ has the value -1 , and $\left|U_{1}\right\rangle_{R},\left|U_{2}\right\rangle_{R},\left|U_{3}\right\rangle_{R}$ are the three vacua at cylinder circumference $R$, normalized to 1 . The numbers $\left\langle U_{i}|\varepsilon(0)| U_{j}\right\rangle_{R}$ can be calculated as described above (see equation (B.2)). It is important, in general, that $\left|U_{1}\right\rangle_{R},\left|U_{2}\right\rangle_{R}$, $\left|U_{3}\right\rangle_{R}$ should be orthogonal, otherwise the inverse of their inner product matrix has to be included in (C.2). In our case the vacua are split at finite $R$ by tunneling effects, so $\left|U_{1}\right\rangle_{R}$, $\left|U_{2}\right\rangle_{R},\left|U_{3}\right\rangle_{R}$ belong to different eigenvalues, therefore they can be identified uniquely and they are orthogonal. 
The problem of the diagonalization of $\left\langle U_{i}|\varepsilon(0)| U_{j}\right\rangle_{R}$ can be simplified by symmetry considerations in our case: two of the three vacua $\left|U_{1}\right\rangle_{R},\left|U_{2}\right\rangle_{R},\left|U_{3}\right\rangle_{R}$ lie in the spin reversal even subspace, the third one lies in the odd subspace. We choose $\left|U_{1}\right\rangle_{R}$ and $\left|U_{2}\right\rangle_{R}$ to be the even vacua and $\left|U_{3}\right\rangle_{R}$ to be the odd one. Due to the spin reversal symmetry of the $\varepsilon$ perturbation, the matrix elements $\left\langle U_{1,2}|\varepsilon(0)| U_{3}\right\rangle_{R}$ are all 0 , therefore the diagonalization problem can be treated separately in the even and odd sectors. The odd vacuum (which becomes $\frac{|+\rangle-|-\rangle}{\sqrt{2}}$ in infinite volume) thus evolves into the odd (possibly metastable) vacuum of the perturbed theory, and the first-order correction to its energy density is given by

$$
\delta \epsilon_{U_{3}}=\lim _{R \rightarrow \infty} 2 \pi g_{2} \lambda_{2} R^{-2 \Delta_{2}}\left\langle U_{3}|\varepsilon(0)| U_{3}\right\rangle_{R}
$$

In the even sector, one has to consider the 2 by 2 matrix $\left\langle U_{1,2}|\varepsilon(0)| U_{1,2}\right\rangle_{R}$. Since the duality $D$ maps the even sector into itself and commutes with the unperturbed Hamiltonian operator in this sector, and $\left|U_{1}\right\rangle_{R}$ and $\left|U_{2}\right\rangle_{R}$ have different energies, $\left|U_{1}\right\rangle_{R}$ and $\left|U_{2}\right\rangle_{R}$ have to be eigenstates of $D$. The eigenvalues can be +1 or -1 , because $D^{2}=1$. We also know that $\varepsilon$ changes sign under duality, therefore $\left\langle U_{1}|\varepsilon(0)| U_{1}\right\rangle_{R}=\left\langle U_{2}|\varepsilon(0)| U_{2}\right\rangle_{R}=0$. $\varepsilon$ is also self-adjoint, so $\left\langle U_{1}|\varepsilon(0)| U_{2}\right\rangle_{R}=\left\langle U_{2}|\varepsilon(0)| U_{1}\right\rangle_{R}^{*}$. This number can be nonzero if the eigenvalues of $\left|U_{1}\right\rangle_{R}$ and $\left|U_{2}\right\rangle_{R}$ with respect to $D$ have opposite sign. We denote the eigenvectors of the matrix $\left\langle U_{1,2}|\varepsilon(0)| U_{1,2}\right\rangle_{R}$ by $\left|V_{+}\right\rangle_{R}$ and $\left|V_{-}\right\rangle_{R}$. The eigenvalues of the matrix $\left\langle U_{1,2}|\varepsilon(0)| U_{1,2}\right\rangle_{R}$ are $\pm\left|\left\langle U_{1}|\varepsilon(0)| U_{2}\right\rangle_{R}\right|$, so the correction to the energy densities of the two vacua $\left|V_{+}\right\rangle_{\infty}$ and $\left|V_{-}\right\rangle_{\infty}$ are

$$
\delta \epsilon_{V_{+}}=\lim _{R \rightarrow \infty} 2 \pi g_{2} \lambda_{2} R^{-2 \Delta_{2}}\left|\left\langle U_{1}|\varepsilon(0)| U_{2}\right\rangle_{R}\right|
$$

and

$$
\delta \epsilon_{V_{-}}=\lim _{R \rightarrow \infty}-2 \pi g_{2} \lambda_{2} R^{-2 \Delta_{2}}\left|\left\langle U_{1}|\varepsilon(0)| U_{2}\right\rangle_{R}\right| .
$$

Here we have chosen $\left|V_{+}\right\rangle_{R}$ to have the positive eigenvalue and $\left|V_{-}\right\rangle_{R}$ to have the negative one. Note that these formulas would not be meaningful without taking absolute values on the right hand side, since $\left|U_{1}\right\rangle_{R}$ and $\left|U_{2}\right\rangle_{R}$ are defined up to arbitrary phase factors.

The analytic result (C.9) described below, the Landau-Ginzburg picture and our numerical calculations of energy spectra all show that one vacuum gets a negative correction and two vacua get the same positive correction as we move into the high temperature phase. It follows then that

$$
\delta \epsilon_{U_{3}}=\delta \epsilon_{V_{+}}=-\delta \epsilon_{V_{-}}
$$

and $\Delta E=2 \delta \epsilon_{U_{3}}$. The direct TCSA calculation of $\delta \epsilon_{U_{3}}$ and $\delta \epsilon_{V_{+}}$confirms the equality $\delta \epsilon_{U_{3}}=\delta \epsilon_{V_{+}}$and yields the result (4.5) for $\Delta E$.

Having discussed the calculation of the energy density corrections, we turn to the description of the relation between the vacua $\left|U_{1}\right\rangle_{\infty},\left|U_{2}\right\rangle_{\infty},\left|U_{3}\right\rangle_{\infty} ;\left|V_{+}\right\rangle_{\infty},\left|V_{-}\right\rangle_{\infty}$ and $|+\rangle$, $|-\rangle,|0\rangle$. Equation (‥6) implies that

$$
\left\langle U_{3}|\varepsilon| U_{3}\right\rangle_{\infty}=\left|\left\langle U_{1}|\varepsilon| U_{2}\right\rangle_{\infty}\right|
$$


and as we mentioned above, we also have

$$
\left\langle U_{1}|\varepsilon| U_{2}\right\rangle_{\infty}=\left\langle U_{2}|\varepsilon| U_{1}\right\rangle_{\infty}^{*}
$$

and all other matrix elements of $\varepsilon$ between $\left|U_{1}\right\rangle_{\infty},\left|U_{2}\right\rangle_{\infty},\left|U_{3}\right\rangle_{\infty}$ are zero. $\left\langle U_{1}|\varepsilon| U_{2}\right\rangle_{\infty}$ can be made real and positive by a phase redefinition of $\left|U_{1}\right\rangle_{\infty}$ or $\left|U_{2}\right\rangle_{\infty}$.

The matrix elements of $\varepsilon$ between the $R=\infty$ eigenvectors $|+\rangle,|-\rangle,|0\rangle$ were calculated exactly in [25, 26]:

$$
\langle+|\varepsilon|+\rangle=\langle-|\varepsilon|-\rangle=-\langle 0|\varepsilon| 0\rangle=\Lambda_{2}>0,
$$

and the nondiagonal matrix elements are 0 (the numerical value of $\Lambda_{2}$ is given by (4.4), but it is irrelevant here). This result, together with the spin reversal properties, (C.7) and (C.8), allows us to identify $\left|U_{1}\right\rangle_{\infty},\left|U_{2}\right\rangle_{\infty},\left|U_{3}\right\rangle_{\infty}$ in terms of $|+\rangle,|-\rangle,|0\rangle$ as follows:

$$
\begin{aligned}
& \left|U_{1}\right\rangle_{\infty}=\alpha \frac{|+\rangle+|-\rangle}{2}+\beta \frac{|0\rangle}{\sqrt{2}} \\
& \left|U_{2}\right\rangle_{\infty}=\alpha \frac{|+\rangle+|-\rangle}{2}-\beta \frac{|0\rangle}{\sqrt{2}} \\
& \left|U_{3}\right\rangle_{\infty}=\gamma \frac{|+\rangle-|-\rangle}{\sqrt{2}},
\end{aligned}
$$

where it is also assumed that the phases of $\left|U_{1}\right\rangle_{\infty}$ and $\left|U_{2}\right\rangle_{\infty}$ are chosen so that $\left\langle U_{1}|\varepsilon| U_{2}\right\rangle_{\infty}$ is real and positive. $\alpha, \beta$ and $\gamma$ are unknown phase factors which are not determined by the information we have described so far, and they can be set to 1 by the following redefinition of the phases of $|+\rangle,|-\rangle,|0\rangle$ and $\left|U_{3}\right\rangle_{R}:|+\rangle \rightarrow \frac{1}{\alpha}|+\rangle,|-\rangle \rightarrow \frac{1}{\alpha}|-\rangle,|0\rangle \rightarrow \frac{1}{\beta}|0\rangle$, $\left|U_{3}\right\rangle_{R} \rightarrow \frac{\gamma}{\alpha}\left|U_{3}\right\rangle_{R}$. These redefinitions leave the previously stated relations involving these vectors unchanged. $\left|V_{+}\right\rangle_{\infty}$ and $\left|V_{-}\right\rangle_{\infty}$ can now be expressed as

$$
\begin{aligned}
& \left|V_{+}\right\rangle_{\infty}=\frac{\left|U_{1}\right\rangle_{\infty}+\left|U_{2}\right\rangle_{\infty}}{\sqrt{2}}=\frac{|+\rangle+|-\rangle}{\sqrt{2}} \\
& \left|V_{-}\right\rangle_{\infty}=\frac{\left|U_{1}\right\rangle_{\infty}-\left|U_{2}\right\rangle_{\infty}}{\sqrt{2}}=|0\rangle .
\end{aligned}
$$

We note finally that since $\left|U_{1}\right\rangle_{\infty}$ and $\left|U_{2}\right\rangle_{\infty}$ are duality eigenstates with eigenvalues of opposite sign, equations (‥10) and (C.11) imply that the duality maps $|0\rangle$ and $\frac{|+\rangle+|-\rangle}{\sqrt{2}}$ into each other.

\section{Appendix D}

\section{Kink form factors}

Along the first-order phase transition line $\left(g_{2}=0, g_{4}<0\right)$, when the TIM has three degenerate vacua and an exact supersymmetry, in the infinite volume $(R=\infty)$ the twokink form factors

$$
\begin{aligned}
\left\langle 0|\varepsilon(0)| K_{0-}\left(\theta_{1}\right) K_{-0}\left(\theta_{2}\right)\right\rangle & \equiv F_{0-}^{\varepsilon}\left(\theta_{1}-\theta_{2}\right), \\
\left\langle 0|\varepsilon(0)| K_{0+}\left(\theta_{1}\right) K_{+0}\left(\theta_{2}\right)\right\rangle & \equiv F_{0+}^{\varepsilon}\left(\theta_{1}-\theta_{2}\right), \\
\left\langle-|\varepsilon(0)| K_{-0}\left(\theta_{1}\right) K_{0-}\left(\theta_{2}\right)\right\rangle & \equiv F_{-0}^{\varepsilon}\left(\theta_{1}-\theta_{2}\right), \\
\left\langle+|\varepsilon(0)| K_{+0}\left(\theta_{1}\right) K_{0+}\left(\theta_{2}\right)\right\rangle & \equiv F_{+0}^{\varepsilon}\left(\theta_{1}-\theta_{2}\right)
\end{aligned}
$$


are the following [23]:

$$
\begin{aligned}
& F_{0-}^{\varepsilon}(\theta)=F_{0+}^{\varepsilon}(\theta), \quad F_{-0}^{\varepsilon}(\theta)=F_{+0}^{\varepsilon}(\theta), \\
& F_{0-}^{\varepsilon}(\theta)=-\mathrm{i}\left(U_{-}-U_{0}\right) \frac{\mathrm{e}^{-\frac{\gamma}{2}(\pi+\mathrm{i} \theta)}}{p \sinh \frac{1}{p}(\theta-\mathrm{i} \pi)} F_{0}(\theta), \\
& F_{-0}^{\varepsilon}(\theta)=\mathrm{i}\left(U_{-}-U_{0}\right) \frac{\mathrm{e}^{\frac{\gamma}{2}(\pi+\mathrm{i} \theta)}}{p \sinh \frac{1}{p}(\theta-\mathrm{i} \pi)} F_{0}(\theta),
\end{aligned}
$$

where

$$
\begin{aligned}
& U_{0}=\langle 0|\varepsilon| 0\rangle, \quad U_{-}=\langle-|\varepsilon|-\rangle=\langle+|\varepsilon|+\rangle, \\
& F_{0}(\theta)=-\mathrm{i} \sinh \frac{\theta}{2} \exp \left[\int_{0}^{\infty} \frac{\mathrm{d} x}{x} \frac{\sinh (1-p) \frac{x}{2}}{\sinh \frac{p x}{2} \cosh \frac{x}{2}} \frac{\sin ^{2}(\mathrm{i} \pi-\theta) \frac{x}{2 \pi}}{\sinh x}\right], \\
& \gamma=\frac{1}{2 \pi} \ln 2, \quad p=4 .
\end{aligned}
$$

These form factors have a pole at i $\pi$, as can be explicitly seen from (D.1), (D.2).

\section{References}

[1] Belavin A, Polyakov A and Zamolodchikov A, 1984 Nucl. Phys. B 241 333; Friedan D, Qiu Z and Shenker S, 1984 Phys. Rev. Lett $\mathbf{5 2} 1575$

[2] Friedan D, Qiu Z, Shenker S, 1985 Phys. Lett. B. 15137

[3] Goddard P, Kent A and Olive D I, 1986 Commun. Math. Phys. 103105

[4] Lukyanov S L and Fateev V A, 1989 Sov. J. Nucl. Phys. 49925 [Yad. Phys. 49 (1989) 1491]

[5] Lassig M, Mussardo G and Cardy J L, 1991 Nucl. Phys. B 348 591-618

[6] Delfino G, Mussardo G and Simonetti P, 1996 Nucl. Phys. B 473469

[7] Delfino G and Mussardo G, 1995 Nucl. Phys. B 455724 hep-th/9507010

[8] Controzzi D and Mussardo G, 2005 Phys. Lett. B 617 133-139 (hep-th/0503018

[9] Mussardo G, 2007 JHEP 0708: 003 arXiv:0706.2546 [hep-th]

[10] Zamolodchikov A B, in "Fields, strings and quantum gravity", Proceedings of the Beijing Conference 1989, edited by Al.B. Zamolodchikov, p. 349. [Nucl. Phys. B 358 (1991) 497]

[11] Zamolodchikov Al B, 1991 Nucl. Phys. B 358524

[12] Kastor D A, Martinec E J and Shenker S H, 1989 Nucl. Phys. B 316590

[13] Christe P and Mussardo G, 1990 Nucl. Phys. B 330465

[14] Fateev V A and Zamolodchikov A B, 1990 Int. J. Mod. Phys. A 51025

[15] Zamolodchikov A B, 1986 Sov. J. Nucl. Phys. 44529

[16] Karowski M and Weisz P, 1978 Nucl. Phys. B 139445

[17] Smirnov F A, Form Factors in Completely Integrable Models of Quantum Field Theory (World Scientific) 1992.

[18] Yurov V P and Zamolodchikov A B, 1990 Int. J. Mod. Phys. A 5 3221-3246

[19] Klassen T R and Melzer E, 1992 Nucl. Phys. B 370 511-550

[20] Fateev V A, 1994 Phys. Lett. B. 32445

[21] Delfino G, Mussardo G and Simonetti P, 1995 Phys. Rev. D 516620

[22] McCoy B M and Wu T T, 1978 Phys. Rev. D 181259

[23] Delfino G, 1999 Nucl. Phys. B 554 537-551 hep-th/9903082

[24] Guida R and Magnoli N, 1997 Phys. Lett. B 411 127-133

[25] Fateev V, Lukyanov S, Zamolodchikov A and Zamolodchikov A, 1998 Nucl. Phys. B 516 652-674 
[26] Fioravanti D, Mussardo G and Simon P, 2001 Phys. Rev. E 63016103 cond-mat/0008216

[27] Acerbi C, Mussardo G and Valleriani A, 1996 Int. J. Mod. Phys. A 11 5327-5364 hep-th/9601113

[28] Pozsgay B and Takács G, 2006 Nucl. Phys. B 748485 hep-th/0604022

[29] Delfino G, Grinza P and Mussardo G, 2006 Nucl. Phys. B 737291 hep-th/0507133

[30] Tóth G Zs, 2004 J. Phys. A 37 9631-9650 hep-th/0406139

[31] Tóth G Zs, "Investigations in two-dimensional quantum field theory by the bootstrap and TCSA methods", PhD thesis, Eötvös University, Budapest, 2006, arXiv:0707.0015[hep-th] 